\title{
Synthesis of steroidal derivatives bearing a small ring using a catalytic [2+2] cycloaddition and a ring-contraction rearrangement
}

\section{$\operatorname{AUTHOR(S):~}$}

Arichi, Norihito; Hata, Kenji; Takemoto, Yoshiji; Yamada, Ken-ichi; Yamaoka, Yousuke; Takasu, Kiyosei

\section{CITATION:}

Arichi, Norihito ...[et al]. Synthesis of steroidal derivatives bearing a small ring using a catalytic [2+2] cycloaddition and a ring-contraction rearrangement. Tetrahedron 2015, $71(2): 233-244$

\section{ISSUE DATE:}

\section{5-01-14}

\section{URL:}

http://hdl.handle.net/2433/243966

\section{RIGHT:}

(c) 2014. This manuscript version is made available under the CC-BY-NC-ND 4.0 license

http://creativecommons.org/licenses/by-nc-nd/4.0/; The full-text file will be made open to the public on 14 January 2017 in accordance with publisher's 'Terms and Conditions for Self-Archiving'.; この論文は出版社版でありません。引 用の際には出版社版をご確認じ利用ください。; This is not the published version. Please cite only the published version. 


\section{Graphical Abstract}

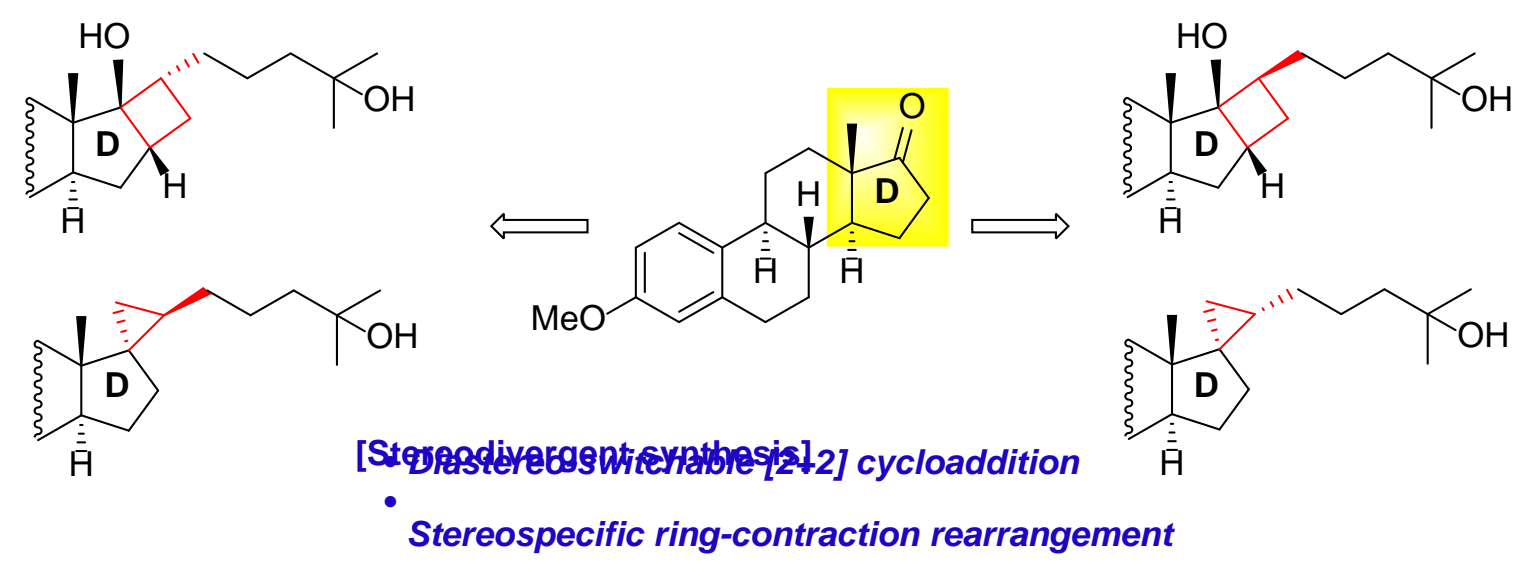

Synthesis of Steroidal Derivatives Bearing a Small Ring Using a Catalytic [2+2] Cycloaddition and a Ring-Contraction Rearrangement

Norihito Arichi, Kenji Hata, Yoshiji Takemoto, Ken-ichi Yamada, Yousuke Yamaoka, and Kiyosei Takasu*

Graduate School of Pharmaceutical Sciences, Kyoto University, Yoshida, Sakyo-ku, Kyoto 606-8501, Japan

E-mail: kay-t@pharm.kyoto-u.ac.jp

Fax: +81 75753 4604; Tel: +81 757534553 


\section{Abstract}

Fixing conformation by introducing a ring structure is a common strategy in drug development. We demonstrate a synthesis that installs a small carbocyclic ring as a structurally-rigid unit in drug lead compounds. Both trans- and cis-cyclobutane rings were constructed in excellent selectivities by controlling the reaction temperature of an $\mathrm{EtAlCl}_{2}$-catalyzed [2+2] cycloaddition between a silyl enol ether and an $\alpha, \beta$-unsaturated ester. Spirocyclopropane rings were stereospecifically formed by our previously reported ring-contraction rearrangement of fused cyclobutanols. This strategy allowed stereodivergent access to a new class of steroidal derivatives bearing a small ring.

\section{Keywords:}

steroids; cyclopropanes; cyclobutanes; [2+2] cycloaddition; ring contraction. 


\section{Introduction}

Rational drug design using protein crystal structures is a rapidly growing area in which several drug discovery success stories have emerged in recent decades. Once the structure of the binding site of the receptor or enzyme is identified, discovery of new small molecule drug leads can be accelerated by using computer simulations such as docking studies. The X-ray crystallographic structure also provides a great opportunity for the optimization of the structure of late-stage leads. For example, the spatial arrangement of the functional groups can be finely tuned at sub-angstrom ( $\AA$ ) resolution in accordance with the crystallographic structure of the binding site. Conformational fixation of flexible drug leads is a popular tactic used to increase activity and/or reduce side effects. A rotatable side chain on the drug scaffold can be restricted by incorporating a structurally rigid moiety such as a double bond, alkyne, amide or aromatic ring. Small-membered carbocycles such as cyclopropane and cyclobutane rings are also used to restrict rotatable side chains in drug development, owing to their structural rigidity. ${ }^{1}$ However, limited numbers of these exist,

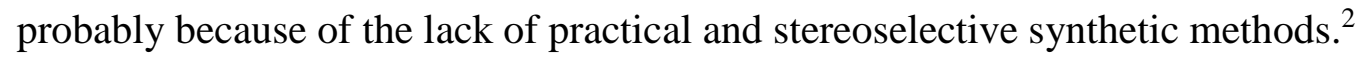

We have reported several unique synthetic methods to construct a cyclobutane ring or a spirocyclopropane ring bearing several functional groups with high stereoselectivity. Catalytic [2+2] cycloaddition of readily available silyl enol ethers and $\alpha, \beta$-unsaturated esters in the presence of an acid 
catalyst, such as $\mathrm{EtAlCl}_{2}$ and triflic imide $\left(\mathrm{Tf}_{2} \mathrm{NH}\right)$, affords functionalized cyclobutanes (Scheme $\left.1 \mathrm{~A}\right){ }^{3}$ The cycloaddition occurs through a stepwise Michael-aldol addition pathway in a diastereoselective manner. A stereospecific ring-contraction rearrangement of fused cyclobutanols to give spirocyclopropanes was also reported (Scheme 1B). ${ }^{4}$ This rearrangement proceeds through a tertiary carbocationic intermediate, which then undergoes rearrangement of the adjacent carbon-carbon bond with release of strain energy.

Scheme 1. Our Methods for Constructing Small Rings

A. Catalytic [2+2] cycloaddition

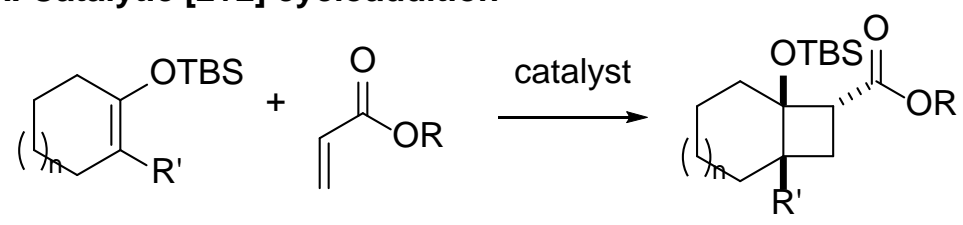

\section{B. Ring-contraction rearrangement}<smiles>[R]C1CC2(O)C3CCCC(C3)C12</smiles>

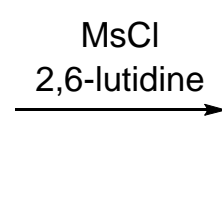<smiles>[R]C1CC12C=CCCC2</smiles>

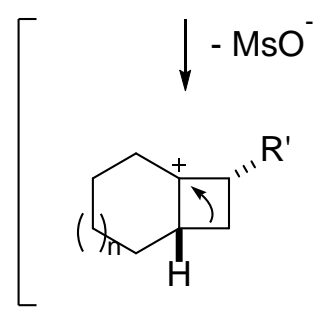<smiles>[R]C1CC12CC1CCCCC12CC</smiles>

We envisaged application of this methodology to the stereoselective synthesis of drug derivatives,

incorporating a cyclobutane ring or a spirocyclopropane moiety to restrict the conformation. A steroidal 
scaffold was chosen as a model drug lead. Steroids are a family of bioactive compounds composed of a unique fused ring system and are widely used as drugs. However, the side effects sometimes become a limiting problem because they have diverse biological activities. For example, calcitriol, which has a steroidal backbone with an opened B-ring, modulates a broad range of biological functions such as bone homeostasis, immunity, cellular growth and differentiation through binding to the vitamin $\mathrm{D}$ receptor. ${ }^{5}$ Although synthetic analogues of calcitriol are effective in the treatment of osteoporosis and psoriasis, ${ }^{6}$ their therapeutic use is limited by severe side effects such as hypercalcemia and hypercalciuria. ${ }^{5 \mathrm{c}}$ Thus, a number of calcitriol analogues have been synthesized to discover which ones display biological activities and reduced side effects better than natural steroid hormones. ${ }^{5 c, 7}$ Mouriño reported calcitriol analogues with a locked side chain by introducing an unsaturated bond or an additional ring. ${ }^{8}$ Some of the analogues exhibited significant biological activities, such as the induction of the transcriptional activity of vitamin $\mathrm{D}$ receptors in human colon cancer cells. ${ }^{8 c}$ Herein, we report a stereodivergent synthesis of steroidal derivatives bearing a small ring using a [2+2] cycloaddition and stereospecific ring-contraction rearrangement. During the course of our synthetic study, switching the diastereoselectivity of the [2+2] cycloaddition depending on the reaction temperature was observed. The mechanistic insight of this is also discussed. ${ }^{9}$ 


\section{Results and discussion}

At the outset of the study, the [2+2] cycloaddition of silyl enol ether 1, prepared from estrone 3-methyl ether, with methyl acrylate (2a) was investigated (Table 1). When silyl enol ether $\mathbf{1}$ was treated with 1.3 equivalents of $\mathbf{2 a}$ at $-78{ }^{\circ} \mathrm{C}$ in the presence of $1.0 \mathrm{~mol} \%$ of $\mathrm{Tf}_{2} \mathrm{NH}$, cyclobutane cis-3a was afforded in only $5 \%$ yield (entry 1). There was a decrease or slight increase in yield when using more equivalents of $\mathrm{Tf}_{2} \mathrm{NH}$ and methyl acrylate (entries 2-4). Reaction with 1,1,3,3-tetrakis(triflyl)propane $\left(\mathrm{Tf}_{2} \mathrm{CHCH}_{2} \mathrm{CHTf}_{2}\right)^{10}$ resulted in no significant improvement in yield (entry 5), whereas $\mathrm{EtAlCl}_{2}$ was an effective catalyst and afforded cis-3a in 77\% yield (entry 6).

Table 1. Catalyst Screening for the [2+2] Cycloaddition of $\mathbf{1}$ with $\mathbf{2 a}$

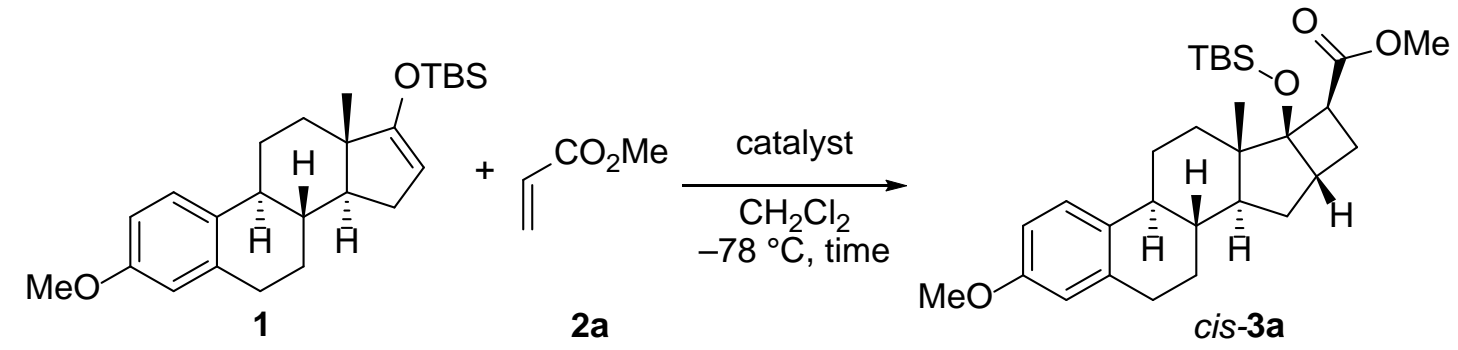

\begin{tabular}{ccccc}
\hline entry & catalyst (mol\%) & 2a (equiv) & time (h) & $\begin{array}{c}\text { yield of } \\
\text { cis-3a(\%) }^{a}\end{array}$ \\
\hline 1 & $\mathrm{Tf}_{2} \mathrm{NH}(1.0)$ & 1.3 & 2.5 & 5 \\
2 & $\mathrm{Tf}_{2} \mathrm{NH}(5.0)$ & 1.3 & 2.5 & 4 \\
3 & $\mathrm{Tf}_{2} \mathrm{NH}(5.0)$ & 2.5 & 1.0 & 33 \\
4 & $\mathrm{Tf}_{2} \mathrm{NH}(20)$ & 2.5 & 2.0 & 10
\end{tabular}




\begin{tabular}{ccccc}
5 & $\mathrm{Tf}_{2} \mathrm{CHCH}_{2} \mathrm{CHTf}_{2}(20)$ & 2.5 & 2.0 & 17 \\
6 & $\mathrm{EtAlCl}_{2}(20)$ & 2.0 & 2.0 & 77 \\
\hline
\end{tabular}

${ }^{a}$ Isolated yields.

Interestingly, when using hexafluoroisopropyl (HFIP) acrylate (2b) instead of 2a, we observed that the diastereoselectivity in the [2+2] cycloaddition switched depending on the reaction conditions (Table 2). Treatment of silyl enol ether $\mathbf{1}$ with $\mathbf{2} \mathbf{b}$ in the presence of $\mathrm{EtAlCl}_{2}$ at $-78{ }^{\circ} \mathrm{C}$ produced trans-3b in $85 \%$ yield with high diastereoselectivity (entry 1). The trans-selectivity remained approximately constant regardless of the reaction time (entries 1 and 2). In contrast, the trans-selectivity of $\mathbf{3 b}$ decreased with an increase in reaction temperature (entries 1, 3-5). When the reaction was carried out at room temperature, cis-3b was obtained exclusively in $74 \%$ yield along with $12 \%$ yield of the Michael adduct 4 (entry 5). The reaction conducted at $-78{ }^{\circ} \mathrm{C}$ for $10 \mathrm{~min}$, followed by stirring at room temperature for an additional 10 min, afforded only the cis-adduct (entry 6). The results clearly indicate that trans-3b is the kinetically favored product whereas cis-3b is the thermodynamically more stable one. Reactions with methyl acrylate (2a) at both room temperature (rt) and $-78{ }^{\circ} \mathrm{C}$ afforded cis-3a exclusively (entry 8 and Table 1 entry 6). Even when the reaction temperature was lowered to $-90{ }^{\circ} \mathrm{C}$, trans-3a was obtained only as a minor diastereomer (entry 7). It appears that the activation energy required for isomerization of trans-3 to cis-3 is dependent on the ester substituent. 
Table 2. Effect of Acrylate and Reaction Temperature in the [2+2] Cycloaddition

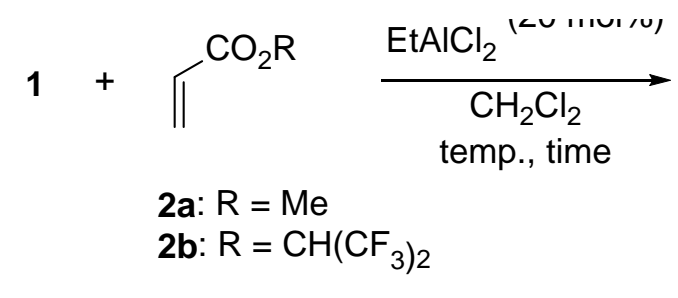

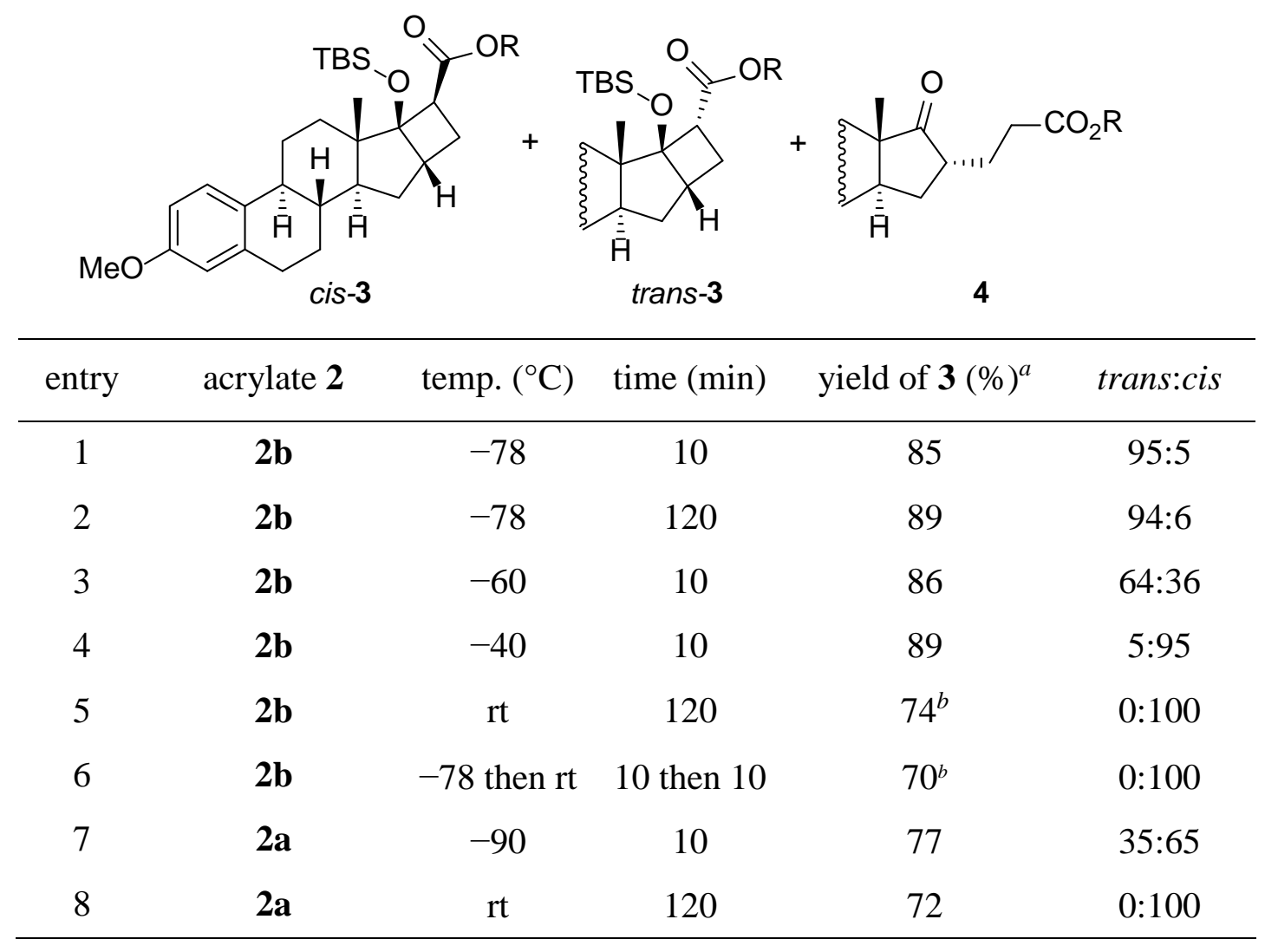

${ }^{a}$ Isolated yields. ${ }^{b}$ Michael adduct 4 was obtained in 5-12 \% yield.

Crossover experiments were performed to investigate the diastereoselectivity switch further.

Treatment of trans-3b with $\mathrm{EtAlCl}_{2}$ at ambient temperature resulted in complete conversion into cis-3b 
(Scheme 2, eq 1), whereas no reaction occurred in the absence of $\mathrm{EtAlCl}_{2}$. Using the same catalyst at the same temperature, cis-3b did not isomerize to trans-3b. When a mixture of the methyl ester cis-3a and an equimolar of HFIP acrylate (2b) was treated with $\mathrm{EtAlCl}_{2}$ at ambient temperature, the crossover adduct cis-3b was afforded in 35\% yield as a single diastereomer, along with 35\% yield of recovered cis-3a (eq 2). These results indicate that $\mathrm{EtAlCl}_{2}$ promotes the retro [2+2] process to reproduce silyl enol ether $\mathbf{1}$ and acrylate 2. HFIP acrylate (2b) competes with in situ generated methyl acrylate (2a) in the [2+2] cycloaddition of the regenerated silyl enol ether $\mathbf{1}$, which results in the isolation of a mixture of cis-3a and cis-3b. In contrast, the reaction of methyl ester cis-3a at $-40{ }^{\circ} \mathrm{C}$ did not provide the crossover adduct, but rather the ring-opened product $\mathbf{4 a}$ in $30 \%$ yield (eq 3). In the reaction of a mixture of trans- and cis-3a at $-40{ }^{\circ} \mathrm{C}$, epimerization of trans-3a into cis-3a occurred along with the formation of $\mathbf{4 a}$, but no crossover product was observed (eq 4).

Scheme 2. Mechanistic Insights into the $\mathrm{EtAlCl}_{2}$-Catalyzed [2+2] Cycloaddition 

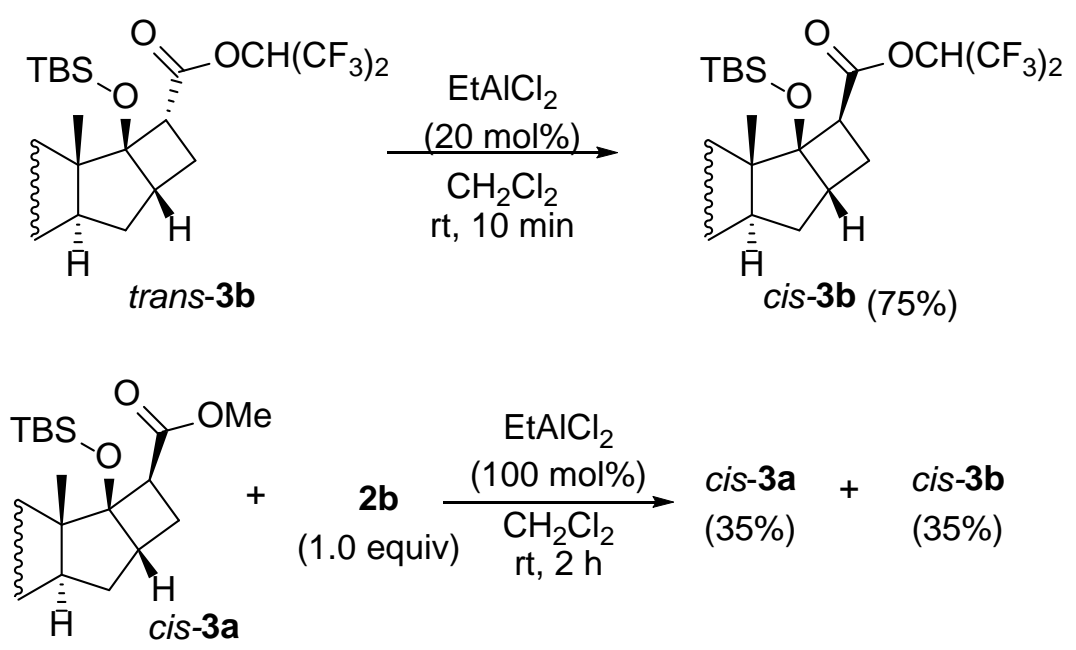

$$
\begin{aligned}
& \mathrm{EtAlCl}_{2}
\end{aligned}
$$

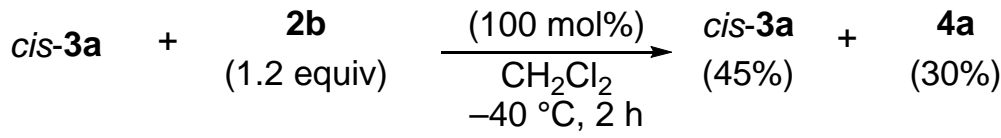

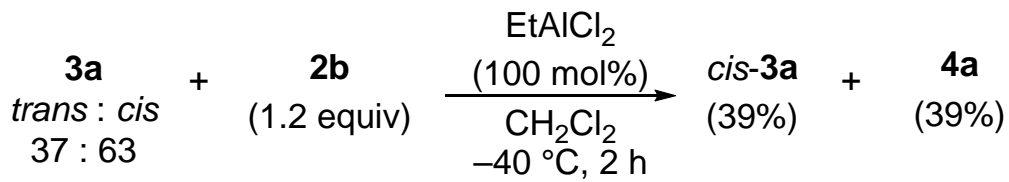

Based on the above results, a possible mechanism for the $\mathrm{EtAlCl}_{2}$-catalyzed [2+2] cycloaddition of $\mathbf{1}$ is shown in Figure 1. Michael addition of silyl enol ether $\mathbf{1}$ to acrylate $\mathbf{2}$ proceeds predominantly at the less hindered $\alpha$-face to give zwitterionic intermediate 5. At lower temperatures, intramolecular aldol-type addition of $\mathbf{5}$ takes place via the preferred transition state TS-I to give trans-3, whereas less favored TS-II affords cis-3 because of steric effects. The product trans-3 would be less stable than cis-3 because of steric repulsion between the axial hydrogen atom at the C14 position and the ester moiety, which is supported by DFT calculations of cis- and trans-3b $\left(\Delta \mathrm{G}^{\circ}{ }_{\text {cis-trans }}=-12.6 \mathrm{~kJ} / \mathrm{mol}\right) .{ }^{11}$ The epimerization process is rather 
complicated. At ambient temperature, trans- and cis-3 equilibrate through a retro [2+2] cycloaddition via silyl enol ether $\mathbf{1}$ to provide the thermodynamically more stable cis-3. In contrast, the retro [2+2] cycloaddition does not proceed at $-40{ }^{\circ} \mathrm{C}$, which indicates that the epimerization to cis-3 at this temperature occurs via siloxonium ion $\mathbf{5}$ through a retro aldol reaction. In addition, ketone $\mathbf{4}$ was presumably formed by desilylation of ketene silyl acetal 6 during work-up.

Figure 1. Possible Mechanism for the $\mathrm{EtAlCl}_{2}$-Catalyzed [2+2] Cycloaddition under Kinetic and Thermodynamic Conditions 


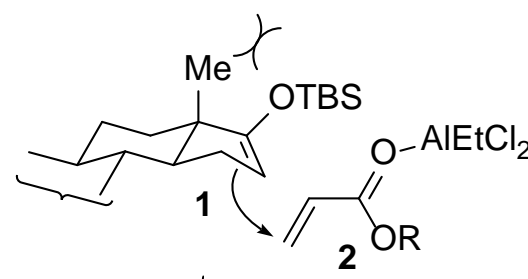

rt $\|-90^{\circ} \mathrm{C}$<smiles>[R]OC(=O)CC[C@H]1C[C@H]2C3CC[C@@H](CC[C@]32C)C1=O</smiles>

work up $\uparrow$

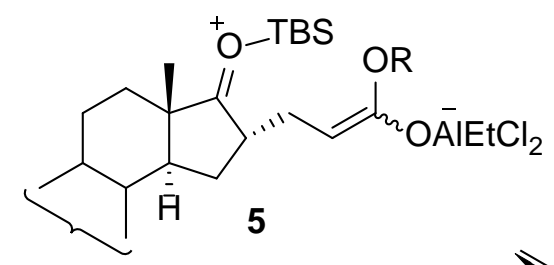

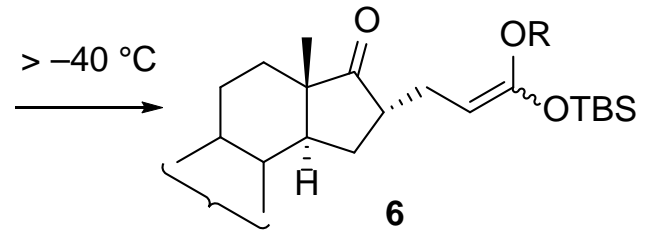

$\begin{aligned} & -90{ }^{\circ} \mathrm{C} \text { for } 2 \mathbf{a} \\ & -60^{\circ} \mathrm{C} \text { for } 2 \mathbf{b}\end{aligned} \|-90^{\circ} \mathrm{C}$

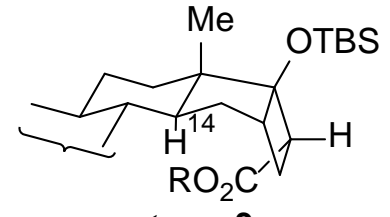

trans-3

kinetically favorable

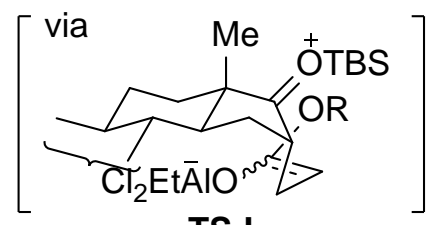

TS-I

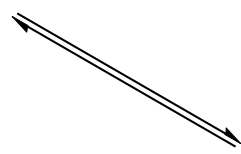

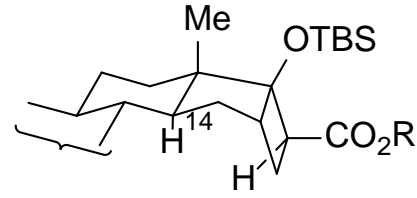

cis-3

\section{thermodynamically favorable}

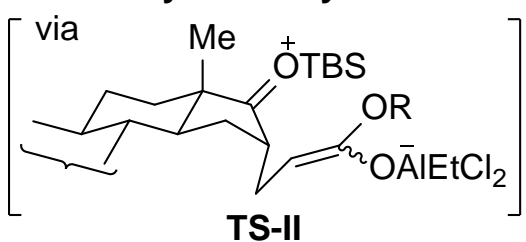

With the cyclobutane scaffolds constructed, we next investigated the ring-contraction

rearrangement of cyclobutanols to give spirocyclopropanes (Scheme 3). To prepare the substrate for the ring-contraction rearrangement, trans-3b was treated with Red-Al to afford cyclobutanol trans-7 in $89 \%$ yield. Removal of the tert-butyldimethylsilyl (TBS) group on the tertiary hydroxyl function followed by benzoyl protection of the primary hydroxyl group furnished trans-9. Cis-9 was also prepared through the same procedure from cis-3b. With the substrates $\mathbf{9}$ for the ring contraction reaction in hands, the reaction 
conditions was firstly screened by using cis-9. According as our previous conditions, ${ }^{4}$ cis-9 $\mathbf{9}$ was treated with methanesulfonyl chloride (MsCl) in 2,6-lutidine, which resulted in the formation of the mesylated product (ca. 30\%), along with recovered starting material (ca. 40\%). We tried other conditions such as oxalyl chloride/triethylamine and triflic anhydride/triethylamine to convert the tertiary hydroxyl group to a good leaving group; however, these attempts proved unsuccessful, and no spirocyclopropane product was observed. The ring contraction rearrangement of cis-9 was finally achieved by using thionyl chloride/triethylamine to afford (20S)-10 in $87 \%$ yield $(16 \beta: 16 \alpha=4: 1)$, the relative configuration was determined by the NOE Spectroscopy. The same conditions were successfully applied to the rearrangement of trans-9 to afford (20R)-10 in a stereospecific manner, albeit in lower yield $(66 \%, 16 \beta: 16 \alpha=7: 3)$. In these reactions, the carbocationic intermediates predominantly undergo nucleophilic attack by the chloride ion to afford (20S)- or (20R)-10, and no elimination products are observed. The difference in yield between (20S)- and (20R)-10 was due to the formation of byproduct 11. We do not fully understand why the ring-contraction rearrangement of trans-9 yielded this unusual product, but it may be influenced by the dihedral angle between the migrating carbon-carbon bond and the empty p-orbital of the carbocationic intermediate.

Scheme 3. Construction of a Spirocyclopropane Ring by Ring-Contraction Rearrangement 

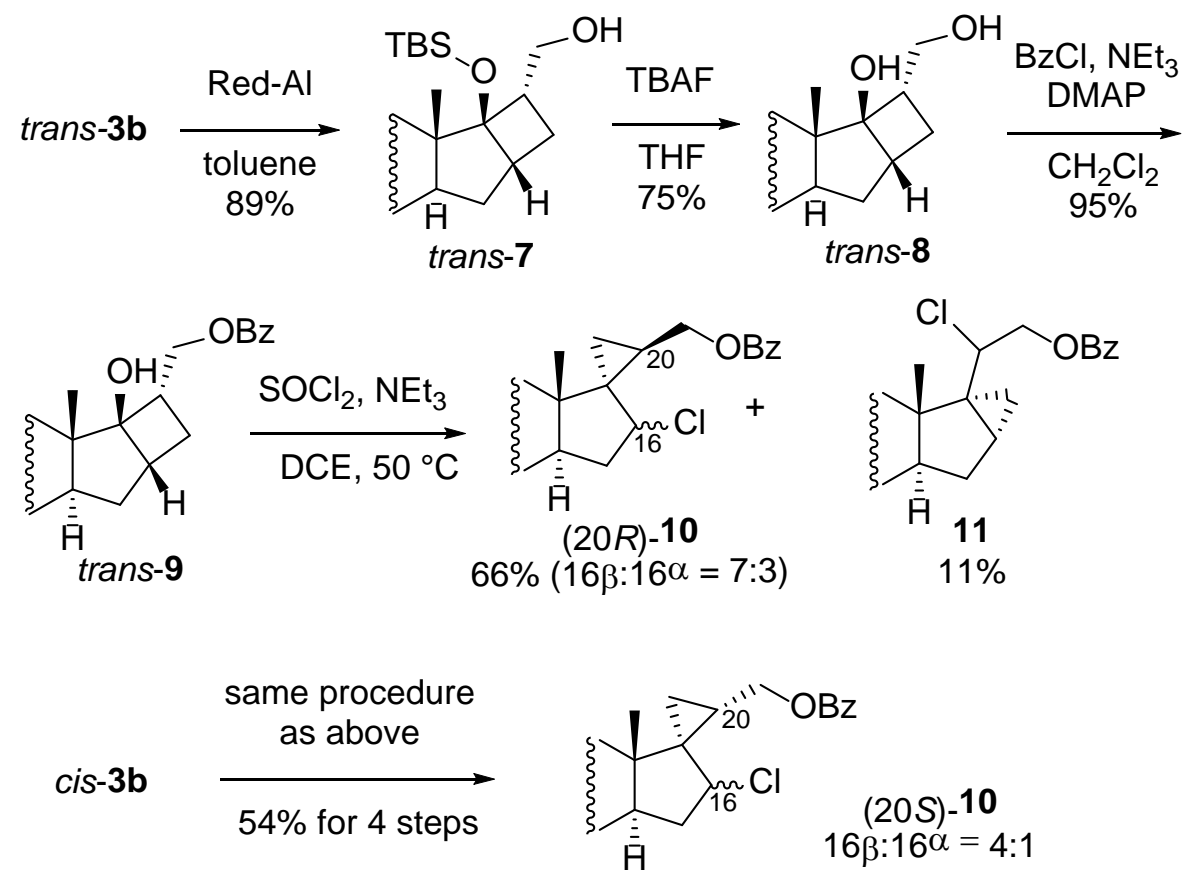

With the estrone derivatives bearing a small ring in hand, our attention turned toward the installation of a cholestane side chain (Scheme 4). Initially, removal of the benzoyl group and dechlorination of (20S)-10 were performed in one-pot using Red-Al to give cyclopropanol (20S)-12, which was then subjected to Swern oxidation to afford aldehyde (20S)-13. Extension of the carbon chain was accomplished by one-pot Julia olefination of the aldehyde using benzothiazolyl sulfone 15, which was prepared from 3-methyl-1,3-butanediol in five steps (Scheme 5). Triethylsilyl (TES) protection of $\mathbf{1 5}$ was necessary for complete conversion of the starting aldehydes in the olefination reactions. Reduction of olefin $\mathbf{1 6}$ was unsuccessfully attempted by hydrogenation using $\mathrm{Pd} / \mathrm{C}$ in ethyl acetate, which resulted in simultaneous ring-opening of the cyclopropane. Accordingly, several catalysts were tested for the chemoselective 
hydrogenation. Reduction with $\mathrm{Pd} / \mathrm{Fib}$ or $\mathrm{Pd} / \mathrm{C}($ en) gave a cyclopropane ring-opened product with the intact carbon-carbon double bond. No reaction occurred using $\mathrm{Pd} / \mathrm{MS} \mathrm{A}^{13}$ or $\mathrm{Pd} / \mathrm{BN}^{14}$ in methanol. In the reaction with $\mathrm{Rh} /$ alumina in ethyl acetate, the double bond was reduced without ring-opening of the cyclopropane, but the aromatic A-ring was also reduced to a cyclohexane ring. Although these efforts using heterogeneous catalysts were unfruitful, chemoselective reduction of the olefin was achieved by diimide reduction, ${ }^{15}$ which was followed by removal of the TES group to give (20S)-17. The final deprotection of the methyl group was troublesome. Treatment with $\mathrm{BBr}_{3}$ or reagents such as TMSI, NaSEt, or $\mathrm{AlMe}_{3} / \mathrm{NaI}^{16}$ gave a complex mixture, probably due to instability of the spirocyclopropane. Nevertheless, the deprotection was accomplished by using $n$-BuLi/ $\mathrm{PPh}_{2} \mathrm{H}^{17}$ to give the final product (20S)-18 in 76\% yield. (20R)-18 was also synthesized by the similar procedure. It is worth noting that treatment of (20R)-10 with Red-Al gave a considerable amount of byproducts. Reduction with sodium borohydride in DMSO at $130{ }^{\circ} \mathrm{C}$ gave $(20 R)-12$ in $53 \%$ yield with less byproduct formation. ${ }^{12}$

Scheme 4. Installation of a Side Chain and Completion of the Synthesis of (20S)- and (20R)-18 

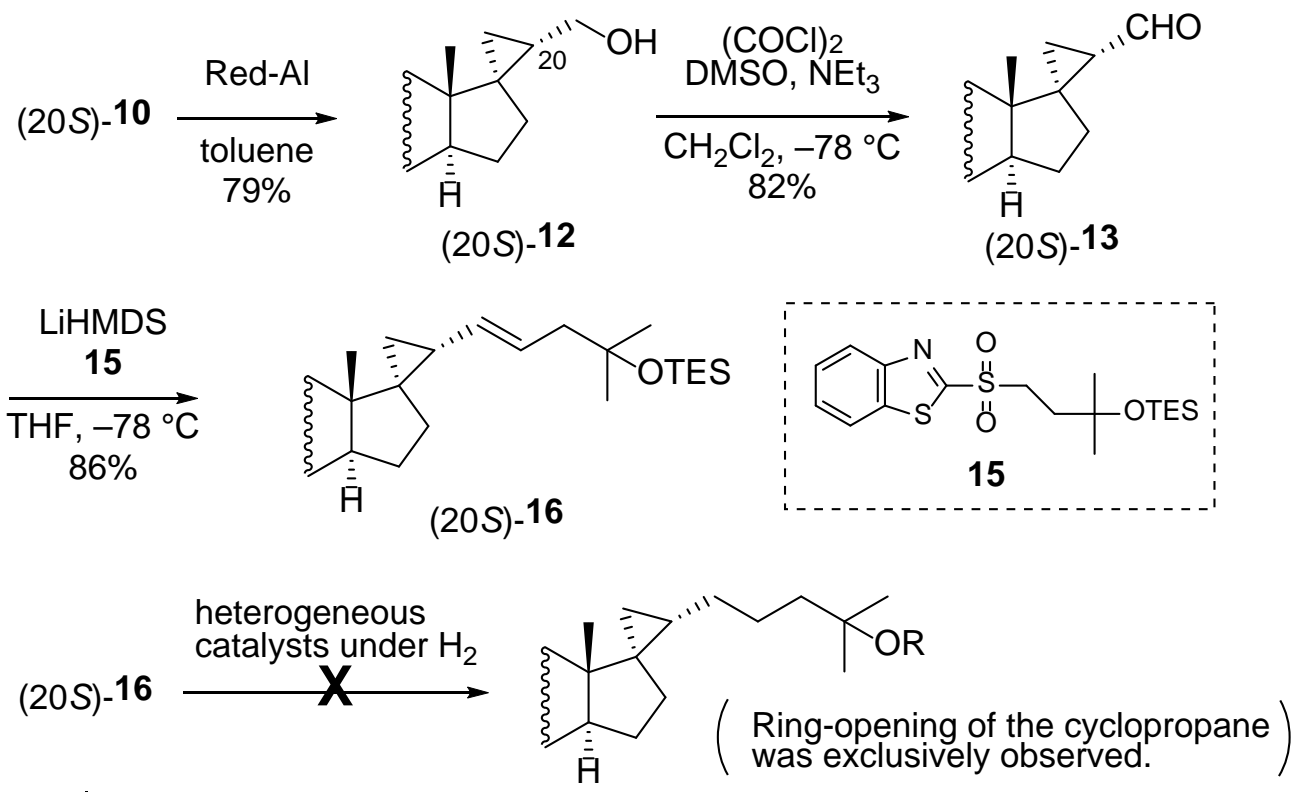
1) $\mathrm{KO}_{2} \mathrm{CN}=\mathrm{NCO}_{2} \mathrm{~K}, \mathrm{AcOH}$ $\mathrm{CH}_{2} \mathrm{Cl}_{2}$, reflux
2) TBAF, THF

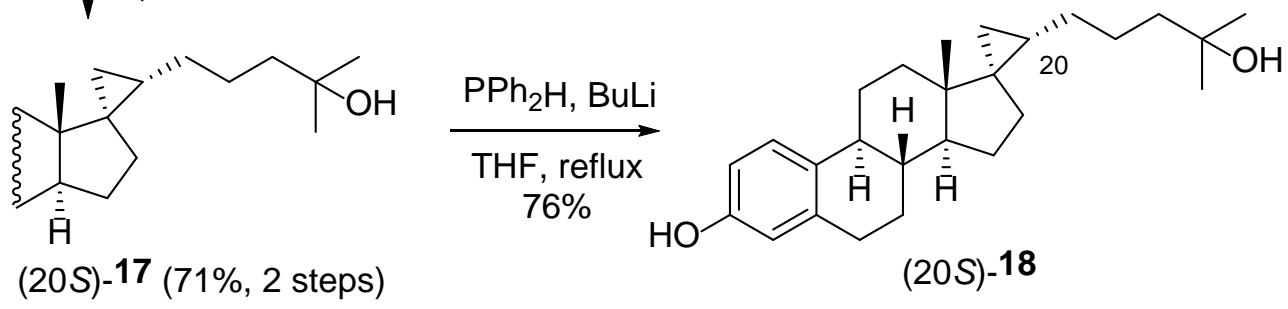

Scheme 5. Preparation of Benzothiazolyl Sulfone 15 


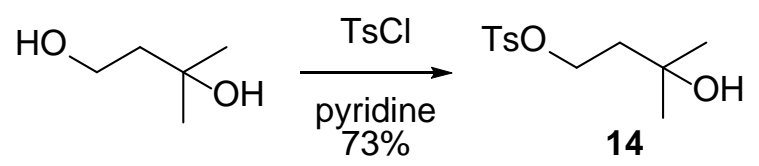

1) Nal, acetone, reflux

2)<smiles></smiles>

$\mathrm{NEt}_{3}, \mathrm{THF}$, reflux

3) $m \mathrm{CPBA}, \mathrm{CH}_{2} \mathrm{Cl}_{2}$

4) TESOTf, $\mathrm{NEt}_{3}, \mathrm{CH}_{2} \mathrm{Cl}_{2}$

Installation of the side chain onto the cyclobutane ring was also accomplished using a similar

strategy (Scheme 6). Swern oxidation followed by one-pot Julia olefination of trans- and cis-7 afforded

cyclobutanes trans- and cis-19, respectively. Reduction of the resulting olefin and removal of the TES group

were carried out in one-pot by hydrogenation with palladium on carbon. Cleavage of the methyl and TBS

groups completed the synthesis of trans- and cis-22, and the structures were verified by X-ray crystallography.

Scheme 6. Installation of a Side Chain and Completion of the Synthesis of trans- and cis-22 

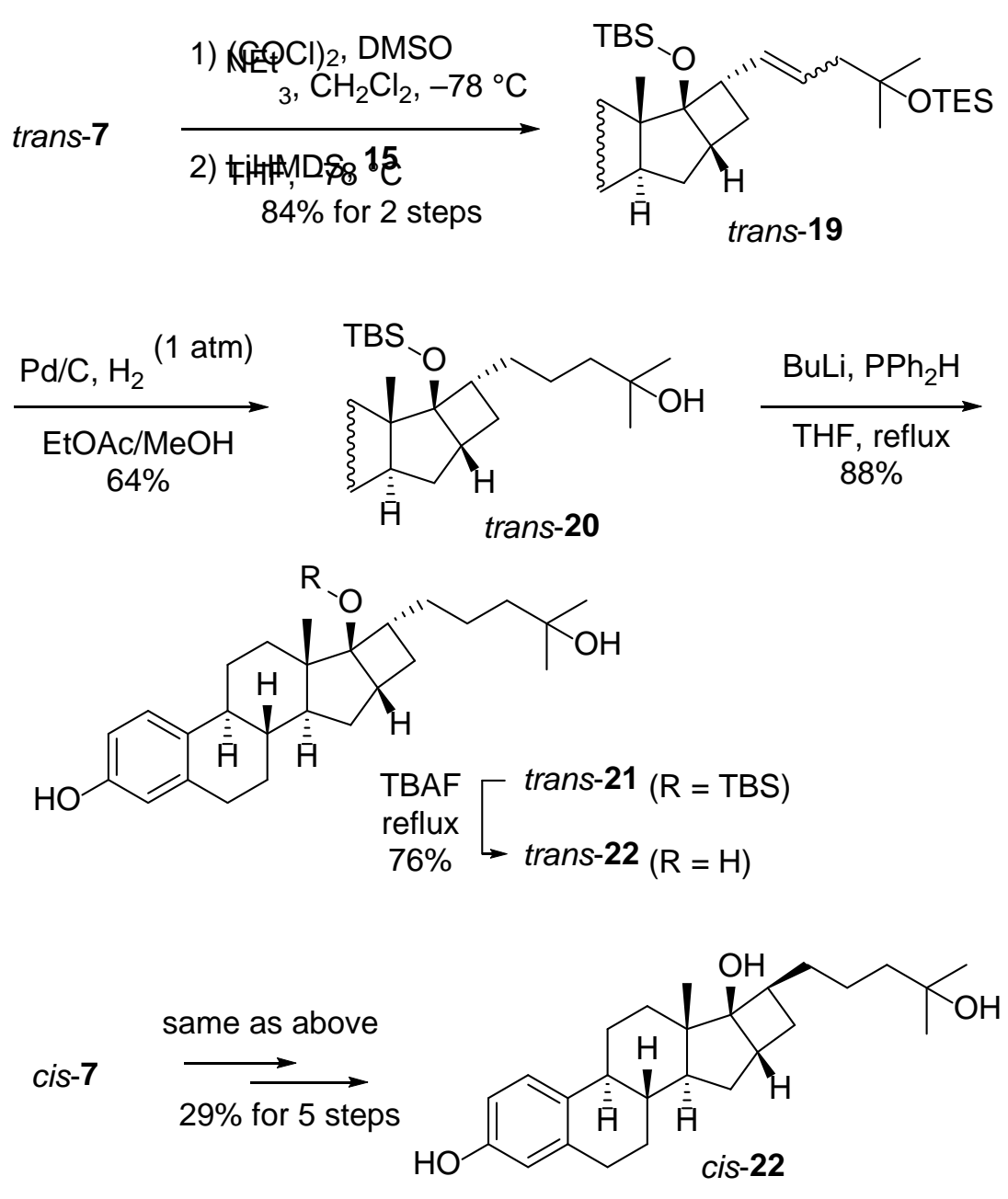

\section{Conclusion}

We have demonstrated the synthesis of four cholestane-type steroidal compounds bearing a cyclobutane or a spirocyclopropane skeleton. The described route features a diastereo-switchable [2+2] cycloaddition reaction and a stereospecific ring-contraction to construct small rings in a stereo-divergent manner. The diastereoselectivity in the [2+2] cycloaddition was inverted under kinetic or thermodynamic conditions. A carbon side chain was installed on the small rings using a one-pot Julia olefination followed by reduction of 
the resulting olefin. This strategy provides a facile route to access a new class of steroid derivatives bearing a small ring. Biological evaluation of the synthetic steroids is currently underway.

\section{Experimental Section}

\subsection{General Remarks}

All non-aqueous reactions were carried out under a positive atmosphere of argon in dried glassware. Dehydrated solvents were purchased for the reactions and used without further desiccation. Reagents were purchased and used without further purification, unless otherwise stated. Reactions were monitored by thin-layer chromatography (TLC) carried out on Merck TLC silica gel $60 \mathrm{~F}_{254}$. Column chromatography was performed using Fuji Silysia BW-200 silica gel. Nuclear magnetic resonance (NMR) spectra were recorded on a JEOL JNM-ECA 500 instrument. The ${ }^{1} \mathrm{H}$ chemical shifts were calibrated with internal tetramethylsilane (TMS, $0 \mathrm{ppm})$ in deuterated organic solvents. The ${ }^{13} \mathrm{C}$ chemical shifts are reported relative to $\mathrm{CDCl}_{3}(77.0$ ppm), DMSO- $d_{6}\left(39.5\right.$ ppm), acetone- $d_{6}\left(206.7\right.$ and 30.4 ppm), MeOH- $d_{4}\left(49.0\right.$ ppm) or THF- $d_{8}$ (67.4 and $25.3 \mathrm{ppm})$. The following abbreviations were used to explain NMR peak multiplicities: $\mathrm{s}=$ singlet, $\mathrm{d}=$ doublet, $\mathrm{t}=$ triplet, $\mathrm{q}=$ quartet, $\mathrm{m}=$ multiplet, $\mathrm{br}=$ broad. Low-resolution mass spectra (LRMS) were recorded on a SHIMADZU GCMS-QP2010 SE spectrometer (EI) or a JEOL MS700 spectrometer (FAB). 
High-resolution mass spectra (HRMS) were recorded on a JEOL MS700 spectrometer (FAB) or a SHIMADZU LCMS-IT-TOF fitted with an ESI. IR experiments were recorded on a SHIMADZU IRAffinity-1 spectrometer. The wave numbers of maximum absorption peaks of IR spectroscopy are presented in $\mathrm{cm}^{-1}$. All melting points were determined using a Yamato MP-21 melting point apparatus and are uncorrected. Optical rotations were obtained on a JASCO P-1030 polarimeter. X-ray diffraction data were recorded on a RIGAKU R-AXIS RAPID system. Compounds $\mathbf{1},{ }^{9}$ cis-3a, ${ }^{9}$ trans- $\mathbf{3 b},{ }^{9}$ cis-3b, ${ }^{9} \mathbf{4 a}^{9}$ and dipotassium azodicarboxylate ${ }^{18}$ were prepared according to previous procedures.

\subsection{Experimental Procedures}


$\mathrm{Na}_{2} \mathrm{SO}_{4}$. Concentration under reduced pressure gave a white solid, which was purified by column chromatography (hexane/EtOAc 9:1) to afford trans-7 (12.4 g, 80\%) as a white solid along with cis-7 (1.39 g, 9\%). Analytical sample was obtained by recrystallization from hexane/EtOAc as white blocks; Found: C, 73.38; H, 9.93. $\mathrm{C}_{28} \mathrm{H}_{44} \mathrm{O}_{3} \mathrm{Si}$ requires C, 73.63; $\mathrm{H}, 9.71 \%$; $\mathrm{Mp} 189-191^{\circ} \mathrm{C} ;[\alpha]_{\mathrm{D}}^{20}+14.2$ (c 1.00, $\left.\mathrm{CHCl}_{3}\right)$; $v_{\max }$ ( $\left.\mathrm{CHCl}_{3}\right) 3494$ (br), 2949, 2855, $1250 \mathrm{~cm}^{-1}$; $\delta_{\mathrm{H}}\left(500 \mathrm{MHz}, \mathrm{CDC1}_{3}\right) 7.21$ (d, $\left.J=8.6 \mathrm{~Hz}, 1 \mathrm{H}\right), 6.72$ (dd, $J=8.6$, $2.6 \mathrm{~Hz}, 1 \mathrm{H}$ ), 6.64 (d, $J=2.6 \mathrm{~Hz}, 1 \mathrm{H}), 4.01$ (ddd, $J=10.0,10.0,6.0 \mathrm{~Hz}, 1 \mathrm{H}$ ), 3.86 (ddd, $J=9.7,9.7,5.8 \mathrm{~Hz}$, 1H), 3.78 (s, 3H), 2.89-2.77 (m, 3H), 2.58-2.51 (m, 1H), 2.36 (ddd, $J=12.0,12.0,9.8 \mathrm{~Hz}, 1 \mathrm{H}$ ), 2.30-2.22 (m, 2H), 1.98-1.89 (m, 1H), 1.81-1.71 (m, 2H), 1.62-1.34 (m, 6H), 1.22 (br s, 1H), 0.97 (ddd, $J$ = 12.9, 8.6, $4.9 \mathrm{~Hz}, 1 \mathrm{H}), 0.90$ (s, 9H), 0.74 (s, 3H), 0.22 (s, 3H), 0.13 (s, 3H); $\delta_{\mathrm{c}}\left(125 \mathrm{MHz}, \mathrm{CDCl}_{3}\right)$ 157.4, 138.0, 132.6, 126.4, 113.8, 111.5, 90.4, 62.7, 55.2, 50.6, 48.3, 47.5, 43.9, 41.6, 38.9, 33.2, 31.9, 29.9, 27.9, 26.5, 25.8, 24.6, 18.2, 17.2, -2.7, -3.1; m/z (EI) 456 (M), 441 (M-Me).

4.2.2. Synthesis

((6bS,8aS,8bR,9R,10aS,11aS,11bR)-8b-((tert-butyldimethylsilyl)oxy)-4-methoxy-8a-methyl-2,6b,7,8,8a,8b,9,1 0,10a,11,11a,11b-dodecahydro-1H-cyclobuta[3,4]cyclopenta[1,2-a]phenanthren-9-yl)methanol (cis-7). To a solution of cis-3b $(8.04 \mathrm{~g}, 13.0 \mathrm{mmol})$ in toluene $(65 \mathrm{~mL})$ was added $3.6 \mathrm{M}$ Red-Al in toluene $(9.0 \mathrm{~mL}, 32$ 
mmol) at $0{ }^{\circ} \mathrm{C}$ under argon. After being stirred for $3 \mathrm{~h}$, the reaction mixture was quenched with saturated aqueous Rochell salt, and stirred vigorously for $30 \mathrm{~min}$. The aqueous layer was extracted with EtOAc, and the combined organic layers were washed with brine, and dried over $\mathrm{Na}_{2} \mathrm{SO}_{4}$. Concentration under reduced pressure gave a white solid, which was purified by column chromatography (hexane/EtOAc 9:1) to afford cis-7 (5.17g, 87\%) as a white solid. Analytical sample was obtained by recrystallization from hexane/EtOAc as white blocks; Found: C, 73.36; H, 9.91. $\mathrm{C}_{28} \mathrm{H}_{44} \mathrm{O}_{3} \mathrm{Si}$ requires C, 73.63; H, 9.71\%; Mp 204-205 ${ }^{\circ} \mathrm{C}$; $[\alpha]_{\mathrm{D}}{ }^{20}$ +29.2 (c 1.00, $\left.\mathrm{CHCl}_{3}\right) ; v_{\max }\left(\mathrm{CHCl}_{3}\right) 3500$ (br), 2929, 2856, $1253 \mathrm{~cm}^{-1} ; \delta_{\mathrm{H}}\left(500 \mathrm{MHz}, \mathrm{CDC1}_{3}\right) 7.22$ (d, $J=$ 8.6 Hz, 1H), 6.72 (dd, $J=8.6,2.6 \mathrm{~Hz}, 1 \mathrm{H}), 6.64$ (d, $J=2.6 \mathrm{~Hz}, 1 \mathrm{H}$ ), 3.83 (ddd, $J=10.9,10.9,2.3 \mathrm{~Hz}, 1 \mathrm{H}$ ), 3.78 (s, 3H), 3.49 (ddd, $J=10.9,10.9,5.8 \mathrm{~Hz}, 1 \mathrm{H}), 2.89-2.86(\mathrm{~m}, 2 \mathrm{H}), 2.78-2.67$ (m, 1H), 2.66-2.59 (m, 1H), 2.41-2.31 (m, 1H), 2.25 (ddd, $J=10.9,10.9,3.4 \mathrm{~Hz}, 1 \mathrm{H}), 1.94-1.83$ (m, 2H), 1.81 (dd, $J=10.9,1.7 \mathrm{~Hz}$, 1H), 1.71-1.59 (m, 3H), 1.53-1.40 (m, 5H), 1.29 (ddd, $J$ = 12.4, 9.2, 2.9 Hz, 1H), 0.94 (s, 9H), 0.73 (s, 3H), 0.30 (s, 3H), 0.16 (s, 3H); $\delta_{\mathrm{C}}\left(125 \mathrm{MHz}, \mathrm{CDCl}_{3}\right)$ 157.4, 137.9, 132.7, 126.4, 113.8, 111.4, 90.7, 63.8, 55.2, 47.9, 46.4, 44.1, 39.3, 38.9, 38.4, 31.9, 29.8, 29.7, 27.7, 26.1, 26.0, 24.2, 18.4, 14.5, -1.2, -2.3; m/z (FAB) $457(\mathrm{M}+\mathrm{H})$. 
(6bS,8aS,8bR,9S,10aS,11aS,11bR)-9-(hydroxymethyl)-4-methoxy-8a-methyl-1,2,6b,7,8,8a,9,10,10a,11,11a,11

b-dodecahydro-8bH-cyclobuta[3,4] cyclopenta[1,2-a]phenanthren-8b-ol (trans-8). A mixture of trans-7 (7.74

g, $16.9 \mathrm{mmol}$ ) and a $1.0 \mathrm{M}$ solution of TBAF in THF ( $51 \mathrm{~mL}, 51 \mathrm{mmol})$ was heated under reflux for $46 \mathrm{~h}$.

After cooling to room temperature, the resulting mixture was diluted with $\mathrm{CHCl}_{3}$, and washed with water.

The aqueous layer was extracted with $\mathrm{CHCl}_{3}$, dried over $\mathrm{Na}_{2} \mathrm{SO}_{4}$. Concentration in vacuo and purification by

column chromatography (EtOAc) gave trans-8 (4.30 g, 75\%) as a pale yellow solid. Analytical sample was

obtained by recrystallization from EtOAc as a white needle; Found: $\mathrm{C}, 77.16 ; \mathrm{H}, 8.83 . \mathrm{C}_{22} \mathrm{H}_{30} \mathrm{O}_{3}$ requires C,

76.88; H, 9.00\%; Mp 199-201 ${ }^{\circ} \mathrm{C}$; $[\alpha]_{\mathrm{D}}^{20}+25.2$ (c 1.00, THF); $v_{\max }\left(\mathrm{CHCl}_{3}\right) 3287$ (br), 2974, 2924, 2859,

1709, 1636, 1501, 1350, 1254, $1184 \mathrm{~cm}^{-1} ; \delta_{\mathrm{H}}\left(500 \mathrm{MHz}, \mathrm{CDC1}_{3}\right) 7.21$ (d, $\left.J=8.9 \mathrm{~Hz}, 1 \mathrm{H}\right), 6.72$ (dd, $J=8.5$,

$2.4 \mathrm{~Hz}, 1 \mathrm{H}$ ), 6.64 (d, $J=2.3 \mathrm{~Hz}, 1 \mathrm{H}), 4.04$ (dd, $J=8.9,8.9 \mathrm{~Hz}, 1 \mathrm{H}$ ), 3.84 (dd, $J=8.6,8.6 \mathrm{~Hz}, 1 \mathrm{H}), 3.78$ (s,

3H), 2.89-2.76 (m, 3H), 2.42-2.25 (m, 4H), 1.96-1.91 (m, 2H), 1.88 (ddd, $J=11.5,11.5,5.7 \mathrm{~Hz}, 1 \mathrm{H}), 1.79$

(ddd, $J=12.0,3.6,3.3 \mathrm{~Hz}, 1 \mathrm{H}$ ), 1.72 (ddd, $J=12.7,12.7,3.9 \mathrm{~Hz}, 1 \mathrm{H}$ ), 1.57-1.36 (m, 6H), 1.03-0.95 (m,

1H), 0.84 (s, 3H); $\delta_{\mathrm{C}}\left(125 \mathrm{MHz}, \mathrm{DMSO}-d_{6}\right)$ 157.0, 137.5, 132.2, 126.2, 113.4, 111.5, 87.2, 60.9, 54.8, 50.2,

47.9, 46.7, 43.4, 41.1, 38.6, 32.7, 31.6, 29.4, 27.4, 26.2, 25.0, 16.4; m/z (EI) 342 (M), 324 (M- $\left.\mathrm{H}_{2} \mathrm{O}\right), 309$ (M-

$\left.\mathrm{H}_{2} \mathrm{O}-\mathrm{Me}\right)$. 
(6bS,8aS,8bR,9R,10aS,11aS,11bR)-9-(hydroxymethyl)-4-methoxy-8a-methyl-1,2,6b,7,8,8a,9,10,10a,11,11a,11

b-dodecahydro-8bH-cyclobuta[3,4]cyclopenta[1,2-a]phenanthren-8b-ol (cis-8). A mixture of cis-7 (34.4 g,

$75.4 \mathrm{mmol}$ ) and a $1.0 \mathrm{M}$ solution of TBAF in THF (226 mL, $226 \mathrm{mmol}$ ) was stirred at room temperature for

$20 \mathrm{~h}$. The resulting mixture was diluted with $\mathrm{CHCl}_{3}$, and washed with water. The aqueous layer was extracted

with $\mathrm{CHCl}_{3}$, dried over $\mathrm{Na}_{2} \mathrm{SO}_{4}$. Concentration in vacuo and purification by column chromatography

(hexane/EtOAc 3:2) gave cis-8 (15.0 g, 83\%) as a white solid. Analytical sample was obtained by

recrystallization from EtOAc as a white needle; Found: C, 77.43; H, 8.89. $\mathrm{C}_{22} \mathrm{H}_{30} \mathrm{O}_{3}$ requires C, 77.16; $\mathrm{H}$,

8.83\%; Mp 203-205 ${ }^{\circ} \mathrm{C}$ (dec.); $[\alpha]_{\mathrm{D}}^{20}+36.5$ (c 1.00, $\mathrm{CHCl}_{3}$ ); $v_{\max }$ (KBr) 3264 (br), 2932, 2920, 2859, 1609,

1499, $1236 \mathrm{~cm}^{-1} ; \delta_{\mathrm{H}}\left(500 \mathrm{MHz}, \mathrm{CDCl}_{3}\right) 7.23(\mathrm{~d}, J=8.6 \mathrm{~Hz}, 1 \mathrm{H}), 6.73(\mathrm{dd}, J=8.6,2.9 \mathrm{~Hz}, 1 \mathrm{H}), 6.64(\mathrm{~d}, J=$

2.6 Hz, 1H), 3.85-3.74 (m, 2H), 3.78 (s, 3H), 2.93-2.82 (m, 2H), 2.62-2.55 (m, 1H), 2.50 (s, 1H), 2.41-2.32

(m, 2H), 2.28-2.20 (m, 2H), 2.17-2.09 (m, 1H), 1.97-1.89 (m, 1H), 1.69 (ddd, $J=11.2,11.2,5.5$ Hz, 1H),

1.56-1.36 (m, 7H), 1.27 (ddd, $J=12.6,9.5,3.5 \mathrm{~Hz}, 1 \mathrm{H}), 0.80$ (s, 3H); $\delta_{\mathrm{C}}\left(125 \mathrm{MHz}\right.$, DMSO-d $\mathrm{d}_{6}$ ) 157.0, 137.4,

132.3, 126.2, 113.4, 111.4, 86.6, 62.3, 54.9, 48.6, 45.4, 43.6, 40.4, 38.3, 37.9, 31.5, 29.4, 29.3, 27.3, 25.8,

24.9, 13.9; m/z (EI) $342(\mathrm{M}), 324\left(\mathrm{M}-\mathrm{H}_{2} \mathrm{O}\right)$. 
((6bS,8aS,8bR,9S,10aS,11aS,11bR)-8b-hydroxy-4-methoxy-8a-methyl-2,6b,7,8,8a,8b,9,10,10a,11,11a,11b-do

decahydro-1H-cyclobuta[3,4]cyclopenta[1,2-a]phenanthren-9-yl)methyl benzoate (trans-9). To a suspension of trans-8 (3.10 g, $9.05 \mathrm{mmol})$ and DMAP (112 mg, $0.917 \mathrm{mmol})$ in $\mathrm{CH}_{2} \mathrm{Cl}_{2}$ (36 ml) were added triethylamine ( $2.5 \mathrm{~mL}, 18 \mathrm{mmol})$ and benzoyl chloride $(1.3 \mathrm{~mL}, 11 \mathrm{mmol})$. After being stirred for $2 \mathrm{~h}$ at room temperature, the reaction mixture was quenched with saturated aqueous $\mathrm{NaHCO}_{3}$. The aqueous layer was extracted with EtOAc, and the combined organic layers were washed with brine, and dried over $\mathrm{Na}_{2} \mathrm{SO}_{4}$, and concentrated in vacuo to give a brown solid. The residue was purified by column chromatography (hexane/EtOAc 4:1 to 1:1) to yield trans-9 (3.85 g, 95\%) as a white solid. Analytical sample was obtained by recrystallization from hexane/EtOAc as a white crystal; Found: C, 77.84; $\mathrm{H}, 7.67 . \mathrm{C}_{29} \mathrm{H}_{34} \mathrm{O}_{4}$ requires C, 78.00; H, 7.67.\%; Mp 168-169 ${ }^{\circ} \mathrm{C} ;[\alpha]_{\mathrm{D}}^{20}+23.3$ (c 1.00, $\left.\mathrm{CHCl}_{3}\right) ; v_{\max }\left(\mathrm{CHCl}_{3}\right) 3491$ (br), 2936, 2866, 1713, 1609, 1501, 1450, 1273, $1219 \mathrm{~cm}^{-1}$; $\delta_{\mathrm{H}}\left(500 \mathrm{MHz} \mathrm{CDCl}_{3}\right) 8.04$ (dd, $\left.J=7.9,1.3 \mathrm{~Hz}, 2 \mathrm{H}\right), 7.56$ (tt, $J=7.5$, $1.3 \mathrm{~Hz}, 1 \mathrm{H}$ ), 7.44 (dd, $J=7.7,7.7 \mathrm{~Hz}, 2 \mathrm{H}), 7.19$ (d, $J=8.6 \mathrm{~Hz}, 1 \mathrm{H}$ ), 6.70 (dd, $J=8.7,2.7 \mathrm{~Hz}, 1 \mathrm{H}), 6.63$ (d, $J$ $=2.6 \mathrm{~Hz}, 1 \mathrm{H}), 4.63(\mathrm{dd}, J=11.2,7.7 \mathrm{~Hz}, 1 \mathrm{H}), 4.58$ (dd, $J=10.9,8.9 \mathrm{~Hz}, 1 \mathrm{H}), 3.78$ (s, 3H), 3.10-3.03 (m, 1H), 2.93-2.83 (m, 2H), 2.49-2.41 (m, 2H), 2.33-2.27 (m, 2H), 1.96-1.91 (m, 2H), 1.85-1.80 (m, 1H), 1.71 (ddd, $J=12.4,12.4,2.3 \mathrm{~Hz}, 1 \mathrm{H}), 1.62-1.56$ (m, 2H), 1.52-1.38 (m, 3H), 1.18-1.10 (m, 1H), 0.86 (s, 3H); $\delta \mathrm{c}$ 
(125 MHz, $\left.\mathrm{CDCl}_{3}\right)$ 166.6, 157.4, 137.8, 132.9, 132.4, 130.2, 129.5, 128.3, 126.3, 113.7, 111.4, 88.5, 64.4, 55.1, 48.7, 47.2, 45.7, 43.6, 42.2, 38.9, 32.7, 31.6, 29.8, 27.7, 26.3, 24.1, 16.1; m/z (EI) 446 (M), 428 (M$\left.\mathrm{H}_{2} \mathrm{O}\right)$.

4.2.6. Synthesis

((6bS,8aS,8bR,9R,10aS,11aS,11bR)-8b-hydroxy-4-methoxy-8a-methyl-2,6b,7,8,8a,8b,9,10,10a,11,11a,11b-do

decahydro-1H-cyclobuta[3,4]cyclopenta[1,2-a]phenanthren-9-yl)methyl benzoate (cis-9). To a suspension of cis-8 (5.32 g, $15.5 \mathrm{mmol})$ and DMAP (191 mg, $1.56 \mathrm{mmol})$ in $\mathrm{CH}_{2} \mathrm{Cl}_{2}(62 \mathrm{~mL})$ were added triethylamine (4.3 $\mathrm{mL}, 31 \mathrm{mmol})$ and benzoyl chloride $(2.2 \mathrm{~mL}, 19 \mathrm{mmol})$. After being stirred for $3 \mathrm{~h}$ at room temperature, the reaction mixture was quenched with saturated aqueous $\mathrm{NaHCO}_{3}$. The aqueous layer was extracted with EtOAc, and the combined organic layers were washed with brine, and dried over $\mathrm{Na}_{2} \mathrm{SO}_{4}$, and concentrated in vacuo to give a brown solid. The residue was purified by column chromatography (hexane/EtOAc 4:1) to yield cis-9 (6.25 g, 90\%) as a white solid. Analytical sample was obtained by recrystallization from hexane/EtOAc as a white crystal; Found C, 77.91; H, 7.81. $\mathrm{C}_{29} \mathrm{H}_{34} \mathrm{O}_{4}$ requires C, 78.00; H, 7.67\%; Mp $126-$ $128^{\circ} \mathrm{C} ;[\alpha]_{\mathrm{D}}^{20}+38.6\left(\mathrm{c} 1.00, \mathrm{CHCl}_{3}\right) ; v_{\max }\left(\mathrm{CHCl}_{3}\right) 3464$ (br), 2932, 2862, 1713, 1605, 1501, 1450, $1277 \mathrm{~cm}^{-}$ 1; $\delta_{\mathrm{H}}\left(500 \mathrm{MHz} \mathrm{CDCl}_{3}\right) 8.03$ (dd, $\left.J=8.3,1.4 \mathrm{~Hz}, 2 \mathrm{H}\right), 7.58$ (tt, $\left.J=7.5,1.4 \mathrm{~Hz}, 1 \mathrm{H}\right), 7.45$ (dd, $J=8.0,8.0 \mathrm{~Hz}$, 
2H), 7.15 (d, $J=8.6 \mathrm{~Hz}, 1 \mathrm{H}), 6.68$ (dd, $J=8.6,2.9 \mathrm{~Hz}, 1 \mathrm{H}), 6.63$ (d, $J=2.9 \mathrm{~Hz}, 1 \mathrm{H}), 4.75$ (dd, $J=11.5,9.8$

Hz, 1H), 4.24 (dd, $J=11.2,4.0 \mathrm{~Hz}, 1 \mathrm{H}), 3.76$ (s, 3H), 2.92-2.77 (m, 3H), 2.43-2.38 (m, 1H), 2.26-2.16 (m,

2H), 2.10-2.02 (m, 1H), 1.95-1.88 (m, 1H), 1.75-1.67 (m, 1H), 1.60 (ddd, $J=12.3,12.3,8.3$ Hz, 1H), 1.55-

1.34 (m, 7H), 0.80 (s, 3H); $\delta_{\mathrm{C}}\left(125 \mathrm{MHz}, \mathrm{CDCl}_{3}\right)$ 167.1, 157.4, 137.9, 133.0, 132.5, 130.1, 129.5, 128.4,

126.2, 113.7, 111.3, 87.1, 65.2, 55.1, 49.7, 46.0, 44.0, 40.0, 38.6, 35.3, 31.4, 29.8, 29.6, 27.6, 26.0, 24.4,

13.8; $m / z$ (EI) 446 (M).

4.2.7.

Synthesis

of

((2'S,8S,9S,13S,14S,16S,17R)-16-chloro-3-methoxy-13-methyl-6,7,8,9,11,12,13,14,15,16-decahydrospiro[cyc

lopenta[a]phenanthrene-17,1'-cyclopropan]-2'-yl)methyl benzoate ((20S)-10). To a solution of cis-9 (6.15 g,

$13.8 \mathrm{mmol})$ in dichloroethane $(69 \mathrm{~mL})$ were added triethylamine $(3.8 \mathrm{~mL}, 28 \mathrm{mmol})$ and thionyl chloride $(2.0$

$\mathrm{mL}, 28 \mathrm{mmol}$ ) under argon. After being stirred at $50^{\circ} \mathrm{C}$ for $2 \mathrm{~h}$, the reaction was quenched with saturated

aqueous $\mathrm{NaHCO}_{3}$. The aqueous layer was extracted with EtOAc, and the combined organic layers were

washed with brine, dried over $\mathrm{Na}_{2} \mathrm{SO}_{4}$, and concentrated in vacuo. The residue was purified by column

chromatography (hexane/diethyl ether 10:1) to afford (20S)-10 (5.60 g, 87\%, dr 4:1) as a white solid. The

diastereomers were partially separated upon careful column chromatography to obtain an analytical sample. 
Data for the major diasteromer: Mp $177-179{ }^{\circ} \mathrm{C} ;[\alpha]_{\mathrm{D}}^{20}+54.7$ (c 1.00, $\left.\mathrm{CHCl}_{3}\right) ; v_{\max }\left(\mathrm{CHCl}_{3}\right) 2932$, 2859, 1717, 1609, 1501, 1450, 1258, 1177, $1111 \mathrm{~cm}^{-1}$; $\delta_{\mathrm{H}}\left(500 \mathrm{MHz} \mathrm{CDCl}_{3}\right) 8.09$ (dd, $\left.J=8.3,1.2 \mathrm{~Hz}, 2 \mathrm{H}\right), 7.56$ (tt, $J=7.5,1.5 \mathrm{~Hz}, 1 \mathrm{H}), 7.45$ (dd, $J=8.1,8.1 \mathrm{~Hz}, 2 \mathrm{H}), 7.19$ (d, $J=8.6 \mathrm{~Hz}, 1 \mathrm{H}), 6.71(\mathrm{dd}, J=8.6,2.9 \mathrm{~Hz}, 1 \mathrm{H})$, $6.64(\mathrm{~d}, J=2.6 \mathrm{~Hz}, 1 \mathrm{H}), 4.74$ (dd, $J=11.7,6.0 \mathrm{~Hz}, 1 \mathrm{H}), 4.44$ (dd, $J=11.5,8.6 \mathrm{~Hz}, 1 \mathrm{H}), 4.32$ (dd, $J=8.3$, $5.2 \mathrm{~Hz}, 1 \mathrm{H}), 3.78$ (s, 3H), 2.94-2.82 (m, 2H), 2.78 (ddd, $J=\mathrm{Hz}, 13.5,8.0,8.0 \mathrm{~Hz}, 1 \mathrm{H}), 2.34-2.26$ (m, 1H), 2.25-2.17 (m, 1H), 2.00 (ddd, $J=13.5,13.5,5.4$ Hz, 1H), 1.93-1.85 (m, 1H), 1.58-1.35 (m, 5H), 1.31-1.22 (m, 2H), 1.17 (s, 3H), 1.08 (ddd, $J=12.9,12.9,4.1 \mathrm{~Hz}, 1 \mathrm{H}), 0.49$ (dd, $J=5.7,5.7 \mathrm{~Hz}, 1 \mathrm{H}) ; \delta_{\mathrm{c}}(125 \mathrm{MHz}$, $\left.\mathrm{CDCl}_{3}\right)$ 166.7, 157.5, 137.8, 132.8, 132.2, 130.6, 129.6, 128.3, 126.2, 113.8, 111.6, 67.0, 66.8, 55.2, 52.5, 43.8, 43.7, 42.4, 39.6, 39.0, 32.8, 29.7, 27.6, 25.7, 19.3, 18.9, 17.7; m/z (EI) 464 (M), 429 (M-Cl), 342 (MBzOH), 307 (M-Cl-BzOH); HRMS (ESI): [M+Na $]^{+}$, found 487.2018. $\mathrm{C}_{29} \mathrm{H}_{33} \mathrm{ClO}_{3} \mathrm{Na}$ requires 487.2010.

4.2.8.

Synthesis

of

((2'R,8S,9S,13S,14S,16S,17R)-16-chloro-3-methoxy-13-methyl-6,7,8,9,11,12,13,14,15,16-decahydrospiro[cyc lopenta[a]phenanthrene-17,1'-cyclopropan]-2'-yl)methyl benzoate ((20R)-10) and 2-chloro-2-((6bS,8aS,8bS,9aS,10aS,10bS)-4-methoxy-8a-methyl-1,6b,7,8,8a,9,9a,10,10a,10b-decahydrocyclo propa[3,4]cyclopenta[1,2-a]phenanthren-8b(2H)-yl)ethyl benzoate (11). To a solution of trans-9 (1.51 g, 
$3.38 \mathrm{mmol})$ in dichloroethane $(17 \mathrm{~mL})$ were added triethylamine $(0.93 \mathrm{~mL}, 6.7 \mathrm{mmol})$ and thionyl chloride (0.49 mL, $6.8 \mathrm{mmol})$ under argon. After being stirred at $50{ }^{\circ} \mathrm{C}$ for $2 \mathrm{~h}$, the reaction was quenched with saturated aqueous $\mathrm{NaHCO}_{3}$. The aqueous layer was extracted with EtOAc, and the combined organic layers were washed with brine, dried over $\mathrm{Na}_{2} \mathrm{SO}_{4}$, and concentrated in vacuo. The residue was purified by column chromatography (hexane/diethyl ether 10:1) to afford (20R)-10 (1.03 g, 66\%, dr 7:3) as a white solid and $\mathbf{1 1}$ (173 mg, 11\%) as a pale yellow oil. The diastereomers were partially separated upon careful column chromatography to obtain an analytical sample. Data for the major diasteromer: Mp $60-63{ }^{\circ} \mathrm{C} ;[\alpha]_{\mathrm{D}}{ }^{20}+29.3$ (c 1.00, $\left.\mathrm{CHCl}_{3}\right) ; v_{\max }\left(\mathrm{CHCl}_{3}\right)$ 2936, 1717, 1609, 1501, 1450, 1273, $1111 \mathrm{~cm}^{-1} ; \delta_{\mathrm{H}}\left(500 \mathrm{MHz}, \mathrm{CDCl}_{3}\right) 8.09$ (d, $J=7.2 \mathrm{~Hz}, 2 \mathrm{H}), 7.57$ (t, $J=6.9 \mathrm{~Hz}, 1 \mathrm{H}), 7.46$ (dd, $J=8.0,8.0 \mathrm{~Hz}, 2 \mathrm{H}), 7.19$ (d, $J=8.6 \mathrm{~Hz}, 1 \mathrm{H}), 6.72$ (dd, $J$ = 8.3, $2.6 \mathrm{~Hz}, 1 \mathrm{H}), 6.64(\mathrm{~d}, J=2.3 \mathrm{~Hz}, 1 \mathrm{H}), 4.51(\mathrm{dd}, J=11.2,8.3 \mathrm{~Hz}, 1 \mathrm{H}), 4.40(\mathrm{dd}, J=11.5,7.5 \mathrm{~Hz}, 1 \mathrm{H})$, 3.96 (dd, $J=8.0,6.3 \mathrm{~Hz}, 1 \mathrm{H}), 3.78$ (s, 3H), 2.94-2.82 (m, 2H), 2.70-2.63 (m, 1H), 2.34-2.21 (m, 2H), 1.981.82 (m, 3H), 1.65 (ddd, $J=11.8,3.2,3.2 \mathrm{~Hz}, 1 \mathrm{H}), 1.58-1.36$ (m, 5H), 1.17 (s, 3H), 1.13 (dd, $J=5.2,5.2 \mathrm{~Hz}$, 1H), 0.79 (dd, $J=9.5,4.9 \mathrm{~Hz}, 1 \mathrm{H}) ; \delta_{\mathrm{C}}\left(125 \mathrm{MHz}, \mathrm{CDCl}_{3}\right)$ 166.6, 157.5, 137.7, 132.9, 132.1, 130.3, 129.6, 128.3, 126.2, 113.7, 111.5, 69.5, 64.8, 55.2, 53.3, 43.6, 43.3, 41.9, 38.5, 38.3, 36.7, 29.6, 27.5, 25.9, 22.7, 19.2, 17.5; m/z (EI) 464 (M), 429 (M-Cl), 342 (M-BzOH), 307 (M-Cl-BzOH); HRMS (ESI): [M+Na] , found 487.2011. $\mathrm{C}_{29} \mathrm{H}_{33} \mathrm{ClO}_{3} \mathrm{Na}$ requires 487.2010. Data for compound 11: $\delta_{\mathrm{H}}\left(500 \mathrm{MHz}, \mathrm{CDCl}_{3}\right) 8.05$ (d, $J=$ 
$7.8 \mathrm{~Hz}, 2 \mathrm{H}), 7.58$ (t, $J=7.5 \mathrm{~Hz}, 1 \mathrm{H}), 7.46$ (dd, $J=7.8,7.8 \mathrm{~Hz}, 2 \mathrm{H}), 7.18(\mathrm{~d}, J=8.6 \mathrm{~Hz}, 1 \mathrm{H}), 6.71$ (dd, $J=$ 8.6, $2.9 \mathrm{~Hz}, 1 \mathrm{H}), 6.62$ (d, $J=2.6 \mathrm{~Hz}, 1 \mathrm{H}), 4.93$ (dd, $J=7.2,7.2 \mathrm{~Hz}, 1 \mathrm{H}), 4.29$ (dd, $J=11.5,7.8 \mathrm{~Hz}, 1 \mathrm{H}), 4.23$

(dd, $J=11.5,6.1 \mathrm{~Hz}, 1 \mathrm{H}$ ), 3.77 (s, 3H), 2.91-2.80 (m, 2H), 2.36-2.29 (m, 1H), 2.21-2.08 (m, 2H), 1.87-1.80

(m, 1H), 1.72-1.64 (m, 2H), 1.55-1.48 (m, 3H), 1.41 (ddd, $J=11.8,11.8,4.0 \mathrm{~Hz}, 1 \mathrm{H}), 1.37-1.30$ (m, 1H), 1.14 (d, $J=6.3,4.0 \mathrm{~Hz}, 1 \mathrm{H}), 1.10-1.04(\mathrm{~m}, 1 \mathrm{H}), 1.01$ (s, 3H), 0.68 (dd, $J=8.3,6.9 \mathrm{~Hz}, 1 \mathrm{H}) ; \delta_{\mathrm{C}}(125 \mathrm{MHz}$, $\left.\mathrm{CDCl}_{3}\right)$ 166.0, 157.5, 137.8, 133.3, 132.7, 129.72, 129.68, 128.5, 126.1, 113.8, 111.4, 66.2, 60.4, 55.2, 45.9, 44.3, 42.9, 39.0, 37.4, 35.0, 29.7, 27.9, 26.6, 26.3, 24.6, 17.7, 10.5; m/z (FAB) 464 (M), 429 (M-Cl), 307 (M-Cl-BzOH); HRMS (ESI): [M+Na] $]^{+}$, found 487.2021. $\mathrm{C}_{29} \mathrm{H}_{33} \mathrm{ClO}_{3} \mathrm{Na}$ requires 487.2010. 
material was purified by column chromatography (hexane/diethyl ether 7:3) to afford (20S)-12 (1.17 g, 79\%) as a white solid; Found: C, 80.68; H, 9.54. $\mathrm{C}_{22} \mathrm{H}_{30} \mathrm{O}_{2}$ requires $\mathrm{C}$, 80.94; $\mathrm{H}, 9.26 \%$; $\mathrm{Mp} 112-113^{\circ} \mathrm{C}$; $[\alpha]_{\mathrm{D}}{ }^{20}$ +27.1 (c 1.00, $\left.\mathrm{CHCl}_{3}\right) ; v_{\max }\left(\mathrm{CHCl}_{3}\right) 3410$ (br), 3009, 2932, 2866, 1609, 1501, 1450, $1219 \mathrm{~cm}^{-1} ; \delta_{\mathrm{H}}(500$ MHz, $\left.\mathrm{CDCl}_{3}\right) 7.19$ (d, $\left.J=8.6 \mathrm{~Hz}, 1 \mathrm{H}\right), 6.70$ (dd, $\left.J=8.6,2.3 \mathrm{~Hz}, 1 \mathrm{H}\right), 6.63$ (d, $\left.J=2.3 \mathrm{~Hz}, 1 \mathrm{H}\right), 3.77$ (s, 3H), 3.64 (br m, 1H), 3.53 (br m, 1H), 2.91-2.82 (m, 2H), 2.28-2.18 (m, 2H), 2.10-2.03 (m, 1H), 1.94-1.87 (m, 2H), 1.50-1.39 (m, 6H), 1.25-1.18 (m, 2H), 1.11 (ddd, $J=12.6,12.6,4.0 \mathrm{~Hz}, 1 \mathrm{H}), 0.99-0.92$ (m, 2H), 0.81 (s, 3H); $\delta_{\mathrm{C}}\left(125 \mathrm{MHz}, \mathrm{CDCl}_{3}\right)$ 157.4, 138.0, 132.8, 126.2, 113.7, 111.4, 65.1, 55.1, 53.3, 43.9, 41.4, 39.5, 36.3, 33.2, 29.9, 29.0, 27.8, 26.1, 24.6, 19.8, 17.2, 16.2; m/z (EI) 326 (M), 308 (M- $\left.\mathrm{H}_{2} \mathrm{O}\right)$.

4.2.10. Synthesis ((2'R,8S,9S,13S,14S,17S)-3-methoxy-13-methyl-6,7,8,9,11,12,13,14,15,16-decahydrospiro[cyclopenta[a]phen anthrene-17,1'-cyclopropan]-2'-yl)methanol ((20R)-12). A mixture of (20R)-10 (1.59 g, $3.42 \mathrm{mmol})$, $\mathrm{NaBH}_{4}$ (776 mg, $20.5 \mathrm{mmol})$ and DMSO $(14 \mathrm{~mL})$ was stirred for $20 \mathrm{~h}$ at $130^{\circ} \mathrm{C}$. After cooling to room temperature, water was added to the mixture. The aqueous layer was extracted with hexane/EtOAc (4:1), and the combined organic layers were washed with brine, dried over $\mathrm{Na}_{2} \mathrm{SO}_{4}$, and concentrated in vacuo. The residue was partially purified by column chromatography (hexane/diethyl ether 1:1) to give (20R)-12 (584 mg, 53\%) 
as a white gum; $[\alpha]_{\mathrm{D}}^{20}+19.6\left(\right.$ c 1.00, $\left.\mathrm{CHCl}_{3}\right) ; v_{\max }\left(\mathrm{CHCl}_{3}\right) 3383$ (br), 2928, 2862, 1609, 1574, 1501, 1454, 1281, $1018 \mathrm{~cm}^{-1} ; \delta_{\mathrm{H}}\left(500 \mathrm{MHz}, \mathrm{CDCl}_{3}\right) 7.19(\mathrm{~d}, J=8.6 \mathrm{~Hz}, 1 \mathrm{H}), 6.71$ (dd, $\left.J=8.3,2.6 \mathrm{~Hz}, 1 \mathrm{H}\right), 6.63(\mathrm{~d}, J=$ $2.6 \mathrm{~Hz}, 1 \mathrm{H}$ ), 3.87 (dd, $J=10.9,6.3 \mathrm{~Hz}, 1 \mathrm{H}), 3.77$ (s, 3H), 3.54 (dd, $J=10.9,10.9 \mathrm{~Hz}, 1 \mathrm{H}), 2.93-2.78$ (m, 2H), 2.31-2.17 (m, 2H), 2.14-2.03 (m, 1H), 1.97-1.89 (m, 1H), 1.88-1.78 (m, 1H), 1.58-1.30 (m, 8H), 1.171.11 (m, 1H), 0.89 (s, 3H), 0.67 (dd, $J=4.6,4.6 \mathrm{~Hz}, 1 \mathrm{H}), 0.42$ (dd, $J=8.3,4.3 \mathrm{~Hz}, 1 \mathrm{H}) ; \delta_{\mathrm{c}}(125 \mathrm{MHz}$, $\left.\mathrm{CDCl}_{3}\right)$ 157.3, 137.9, 132.6, 126.2, 113.7, 111.4, 63.1, 55.1, 54.5, 43.5, 41.4, 39.1, 36.6, 35.8, 29.8, 27.6, 26.4, 25.0, 24.6, 17.1 (two signals missing); $\mathrm{m} / \mathrm{z}$ (EI) 326 (M); HRMS (ESI): [M+Na $]^{+}$, found 349.2145. $\mathrm{C}_{22} \mathrm{H}_{30} \mathrm{O}_{2} \mathrm{Na}$ requires 349.2138 .

4.2.11. Synthesis (2'S,8S,9S,13S,14S,17S)-3-methoxy-13-methyl-6,7,8,9,11,12,13,14,15,16-decahydrospiro[cyclopenta[a]phen anthrene-17,1'-cyclopropane]-2'-carbaldehyde ((20S)-13). To a solution of oxalyl chloride (0.32 mL, 3.7 mmol) in $\mathrm{CH}_{2} \mathrm{Cl}_{2}(4.0 \mathrm{~mL})$ was dropwise added a solution of DMSO $(0.40 \mathrm{~mL}, 5.6 \mathrm{mmol})$ in $\mathrm{CH}_{2} \mathrm{Cl}_{2}(5.0$ $\mathrm{mL})$ at $-78{ }^{\circ} \mathrm{C}$. After being stirred for $5 \mathrm{~min}$, a solution of (20S)-12 (601 mg, $\left.1.84 \mathrm{mmol}\right)$ in $\mathrm{CH}_{2} \mathrm{Cl}_{2}(9.0 \mathrm{~mL})$ was dropwise added. After being stirred for $10 \mathrm{~min}$ at $-78{ }^{\circ} \mathrm{C}$, triethylamine $(1.5 \mathrm{ml}, 10.8 \mathrm{mmol})$ was added. After being stirred for $20 \mathrm{~min}$, the resulting mixture was allowed to warm to ambient temperature, diluted 
with $\mathrm{CHCl}_{3}$, and quenched with saturated aqueous $\mathrm{NH}_{4} \mathrm{Cl}$. The aqueous layer was extracted with $\mathrm{CHCl}_{3}$, and the combined organic layers were washed with brine, dried over $\mathrm{Na}_{2} \mathrm{SO}_{4}$, and concentrated under reduced pressure. The crude material was purified by column chromatography (hexane/EtOAc 10:1) to afford (20S)-13 (490 mg, 82\%) as a white solid. Analytical sample was obtained by recrystallization from hexane/EtOAc as a white needle; Found C, 81.17; H, 8.55. $\mathrm{C}_{22} \mathrm{H}_{28} \mathrm{O}_{2}$ requires C, 81.44; H, 8.70\%; Mp 148$149{ }^{\circ} \mathrm{C}$ (dec.); $[\alpha]_{\mathrm{D}}^{20}+47.7$ (c 1.00, $\left.\mathrm{CHCl}_{3}\right) ; v_{\max }\left(\mathrm{CHCl}_{3}\right) 2932,2913,2866,1701,1609,1501,1238,1172$, $1042 \mathrm{~cm}^{-1} ; \delta_{\mathrm{H}}\left(500 \mathrm{MHz}, \mathrm{CDCl}_{3}\right) 9.12(\mathrm{~d}, J=6.3 \mathrm{~Hz}, 1 \mathrm{H}), 7.19(\mathrm{~d}, J=8.6 \mathrm{~Hz}, 1 \mathrm{H}), 6.71$ (dd, $J=8.6,2.6 \mathrm{~Hz}$, 1H), 6.63 (d, $J=2.3 \mathrm{~Hz}, 1 \mathrm{H}), 3.77$ (s, 3H), 2.97-2.83 (m, 2H), 2.33-2.17 (m, 3H), 2.00-1.93 (m, 2H), 1.791.70 (m, 2H), 1.52-1.39 (m, 6H), 1.26 (ddd, $J=12.1,3.4,3.4 \mathrm{~Hz}, 1 \mathrm{H}), 1.18-1.15$ (m, 2H), 0.83 (s, 3H); $\delta \mathrm{c}$ (125 MHz, $\left.\mathrm{CDCl}_{3}\right)$ 201.8, 157.5, 137.9, 132.3, 126.2, 113.8, 111.4, 55.1, 52.4, 43.80, 43.78, 42.6, 39.3, 32.9, 31.0, 29.8, 29.5, 27.7, 25.9, 24.7, 21.0, 17.1; m/z (EI) 324 (M), 309 (M-Me), 291 (M-Me--H2O).

4.2.12.

Synthesis

(2'R,8S,9S,13S,14S,17S)-3-methoxy-13-methyl-6,7,8,9,11,12,13,14,15,16-decahydrospiro[cyclopenta[a]phen

anthrene-17,1'-cyclopropane]-2'-carbaldehyde ((20R)-13). To a solution of oxalyl chloride (0.37 mL, 4.3 $\mathrm{mmol})$ in $\mathrm{CH}_{2} \mathrm{Cl}_{2}(5.0 \mathrm{~mL})$ was dropwise added a solution of DMSO $(0.46 \mathrm{ml}, 6.5 \mathrm{mmol})$ in $\mathrm{CH}_{2} \mathrm{Cl}_{2}(5.0 \mathrm{~mL})$ 
at $-78{ }^{\circ} \mathrm{C}$. After being stirred for $5 \mathrm{~min}$, a solution of (20R)-12 (584 mg, $\left.1.80 \mathrm{mmol}\right)$ in $\mathrm{CH}_{2} \mathrm{Cl}_{2}(12 \mathrm{~mL})$ was dropwise added. After being stirred for $20 \mathrm{~min}$ at $-78^{\circ} \mathrm{C}$, triethylamine $(1.8 \mathrm{~mL}, 13 \mathrm{mmol})$ was added. After being stirred for $10 \mathrm{~min}$, the resulting mixture was allowed to warm to ambient temperature, diluted with $\mathrm{CHCl}_{3}$, and quenched with saturated aqueous $\mathrm{NH}_{4} \mathrm{Cl}$. The aqueous layer was extracted with $\mathrm{CHCl}_{3}$, and the combined organic layers were washed with brine, dried over $\mathrm{Na}_{2} \mathrm{SO}_{4}$, and concentrated under reduced pressure. The crude material was purified by column chromatography (hexane/EtOAc 10:1) to afford (20R)-13 (471 mg, 81\%) as a white solid; Found C, 81.50; H, 8.92. $\mathrm{C}_{22} \mathrm{H}_{28} \mathrm{O}_{2}$ requires C, 81.44; $\mathrm{H}, 8.70 \%$; Mp 128-129 ${ }^{\circ} \mathrm{C} ;[\alpha]_{\mathrm{D}}^{20}+42.7$ (c 1.00, $\left.\mathrm{CHCl}_{3}\right) ; v_{\max }\left(\mathrm{CHCl}_{3}\right)$ 2932, 2866, 1694, 1609, 1501, 1161, $1038 \mathrm{~cm}^{-1}$; $\delta_{\mathrm{H}}\left(500 \mathrm{MHz}, \mathrm{CDCl}_{3}\right) 9.16(\mathrm{~d}, J=7.2 \mathrm{~Hz}, 1 \mathrm{H}), 7.19(\mathrm{~d}, J=8.6 \mathrm{~Hz}, 1 \mathrm{H}), 6.72(\mathrm{dd}, J=8.5,2.4 \mathrm{~Hz}, 1 \mathrm{H}), 6.64$ (d, $J=2.0 \mathrm{~Hz}, 1 \mathrm{H}$ ), 3.78 (s, 3H), 2.93-2.83 (m, 2H), 2.37-2.15 (m, 3H), 1.95-1.85 (m, 3H), 1.75 (dd, $J=5.2$, $5.2 \mathrm{~Hz}, 1 \mathrm{H}), 1.62-1.37(\mathrm{~m}, 8 \mathrm{H}), 1.10(\mathrm{dd}, J=7.9,5.0 \mathrm{~Hz}, 1 \mathrm{H}), 0.89(\mathrm{~s}, 3 \mathrm{H}) ; \delta_{\mathrm{C}}\left(125 \mathrm{MHz}, \mathrm{CDCl}_{3}\right) 200.4$, 157.5, 137.8, 132.2, 126.2, 113.8, 111.5, 55.2, 54.4, 44.9, 43.4, 42.0, 39.2, 36.3, 36.2, 35.4, 29.8, 27.5, 26.3, 24.4, 21.9, 18.0; m/z (EI) 324 (M), 309 (M-Me), 291 (M-Me- $\left.\mathrm{H}_{2} \mathrm{O}\right)$.

4.2.13. Synthesis of 3-hydroxy-3-methylbutyl 4-methylbenzenesulfonate (14). To a stirred solution of 3-methyl-1,3-butanediol $(4.19 \mathrm{~g}, 40.2 \mathrm{mmol})$ in pyridine $(100 \mathrm{~mL})$ was added tosyl chloride $(9.15 \mathrm{~g}, 48.0$ 
mmol) at $0{ }^{\circ} \mathrm{C}$. After being stirred for $12 \mathrm{~h}$ at room temperature, the reaction mixture was quenched with water. The aqueous layer was extracted with EtOAc. The combined organic layers were washed with saturated aqueous $\mathrm{NaHCO}_{3}$ and brine, dried over $\mathrm{Na}_{2} \mathrm{SO}_{4}$, concentrated in vacuo, and azeotroped with toluene. The resulting pale yellow oil was purified by column chromatography (hexane/EtOAc 3:2 to 1:1) to afford 14 (7.62 g, 73\%) as a colorless oil; $v_{\max }$ (neat) 3518 (br), 2970, 1597, 1465, 1354, $1172 \mathrm{~cm}^{-1} ; \delta_{\mathrm{H}}(500$ MHz, $\left.\mathrm{CDCl}_{3}\right) 7.80$ (d, $\left.J=8.3 \mathrm{~Hz}, 2 \mathrm{H}\right), 7.35$ (d, $\left.J=8.1 \mathrm{~Hz}, 2 \mathrm{H}\right), 4.21$ (t, $\left.J=6.9 \mathrm{~Hz}, 2 \mathrm{H}\right), 2.45$ (s, 3H), 1.86 (t, $J=6.9 \mathrm{~Hz}, 2 \mathrm{H}), 1.22$ (s, 6H); $\delta_{\mathrm{C}}\left(125 \mathrm{MHz}, \mathrm{CDCl}_{3}\right)$ 144.7, 132.7, 129.7, 127.7, 69.3, 67.5, 41.5, 29.4, 21.4; m/z (FAB) $259(\mathrm{M}+\mathrm{H}), 241(\mathrm{M}-\mathrm{OH})$; HRMS (FAB): $[\mathrm{M}+\mathrm{H}]^{+}$, found 259.0975. $\mathrm{C}_{12} \mathrm{H}_{19} \mathrm{O}_{4} \mathrm{~S}$ requires 259.0999.

4.2.14. Synthesis of 2-((3-methyl-3-((triethylsilyl)oxy)butyl)sulfonyl)benzo[d] thiazole (15). To a solution of $14(7.62 \mathrm{~g}, 29.5 \mathrm{mmol})$ in acetone $(98 \mathrm{~mL})$ was added NaI $(11.2 \mathrm{~g}, 74.7 \mathrm{mmol})$. The mixture was heated under reflux for $2 \mathrm{~h}$. The resulting mixture was allowed to cool to room temperature, and concentrated in vacuo. The residue was dissolved in EtOAc, and washed with water. The aqueous layer was extracted with EtOAc, and the combined organic layers were washed with brine, dried over $\mathrm{Na}_{2} \mathrm{SO}_{4}$, and concentrated under reduced pressure to give a brown oil (5.79 g). To a solution of the brown oil (5.79 g) in THF (90 $\mathrm{mL}$ ) were 
added triethylamine $(5.6 \mathrm{~mL}, 41 \mathrm{mmol})$ and 2-mercaptobenzothiazole (5.67 g, $33.9 \mathrm{mmol})$. After being heated under reflux for $12 \mathrm{~h}$, the reaction mixture was allowed to cool to room temperature, diluted with EtOAc ,and washed with water. The aqueous layer was extracted with EtOAc, and the combined organic layers were washed with brine, and dried over $\mathrm{Na}_{2} \mathrm{SO}_{4}$. Concentration under reduced pressure gave a yellow oil (6.87 g). To a solution of the yellow oil $(6.87 \mathrm{~g})$ in $\mathrm{CH}_{2} \mathrm{Cl}_{2}(135 \mathrm{~mL})$ was added $m$ CPBA (75\%, $18.8 \mathrm{~g}$, $81.7 \mathrm{mmol}$ ) at $0{ }^{\circ} \mathrm{C}$. After being stirred for $6 \mathrm{~h}$ at room temperature, the reaction mixture was quenched with saturated aqueous $\mathrm{NaHCO}_{3}$. The organic layer was washed with brine, dried over $\mathrm{Na}_{2} \mathrm{SO}_{4}$, and concentrated in vacuo. The residue was dissolved in $\mathrm{CHCl}_{3}$, and passed through a short pad of silica gel with hexane/EtOAc (1:1) to give a white solid. (7.67 g). To a solution of the white solid (7.67 g) in $\mathrm{CH}_{2} \mathrm{Cl}_{2}(67$ $\mathrm{mL})$ were added triethylamine $(7.5 \mathrm{~mL}, 54 \mathrm{mmol})$ and triethylsilyl trifluoromethanesulfonate $(7.3 \mathrm{~mL}, 32$ mmol) at $0{ }^{\circ} \mathrm{C}$ under argon. After being stirred for $7 \mathrm{~h}$ at room temperature, the reaction mixture was quenched with water. The aqueous layer was extracted with ether, and the combined organic layers were washed with brine, dried over $\mathrm{Na}_{2} \mathrm{SO}_{4}$, and concentrated in vacuo to give a brown solid. The residue was purified by column chromatography (hexane/EtOAc 10:1) to afford $\mathbf{1 5}$ (8.49 g, 72\%, 4 steps) as a white solid; Found C, 53.90; H, 7.31; N, 3.47. $\mathrm{C}_{18} \mathrm{H}_{29} \mathrm{NO}_{3} \mathrm{~S}_{2}$ Si requires C, 54.10; H, 7.31; N, 3.50\%; Mp 57-59 C; $v_{\max }$ (neat) 2951, 2873, 1470, 1327, 1304, $1146 \mathrm{~cm}^{-1} ; \delta_{\mathrm{H}}\left(500 \mathrm{MHz}, \mathrm{CDCl}_{3}\right) 8.23$ (dd, $\left.J=8.2,1.4 \mathrm{~Hz}, 1 \mathrm{H}\right)$, 
8.02 (dd, $J=8.1,1.4 \mathrm{~Hz}, 1 \mathrm{H}), 7.65$ (ddd, $J=8.2,7.0,1.4 \mathrm{~Hz}, 1 \mathrm{H}$ ), 7.60 (ddd, $J=7.8,6.6,1.5 \mathrm{~Hz}, 1 \mathrm{H}$ ), 3.65-

$3.62(\mathrm{~m}, 2 \mathrm{H}), 1.95-1.92(\mathrm{~m}, 2 \mathrm{H}), 1.23(\mathrm{~s}, 6 \mathrm{H}), 0.87(\mathrm{t}, J=8.0 \mathrm{~Hz}, 9 \mathrm{H}), 0.51$ (q, $J=8.0 \mathrm{~Hz}, 6 \mathrm{H}) ; \delta_{\mathrm{C}}(125$

MHz, $\mathrm{CDCl}_{3}$ ) 65.6, 152.7, 136.7, 127.9, 127.5, 125.4, 122.2, 71.8, 51.2, 36.9, 29.7, 6.9, 6.5; m/z (FAB) 400

(M+H), 370 (M-Et), 268 (M-TES).

4.2.15.

Synthesis

of

triethyl(((E)-5-((2'R,8S,9S,13S,14S,17S)-3-methoxy-13-methyl-6,7,8,9,11,12,13,14,15,16-decahydrospiro[cyc

lopenta[a]phenanthrene-17,1'-cyclopropan]-2'-yl)-2-methylpent-4-en-2-yl)oxy)silane ((20S)-16). To a

solution of 15 (352 mg, $1.08 \mathrm{mmol})$ in THF (6.0 mL) was added a 1.0 M solution of LiHMDS in toluene (1.6

$\mathrm{mL}, 1.6 \mathrm{mmol}$ ) at $-78^{\circ} \mathrm{C}$ under argon. After being stirred for $30 \mathrm{~min}$, a solution of (20S)-13 (352 mg, 1.08

mmol) in THF (10 mL) was added, and stirred for $1 \mathrm{~h}$. The reaction mixture was diluted with EtOAc, and

quenched with saturated aqueous $\mathrm{NH}_{4} \mathrm{Cl}$. The aqueous layer was extracted with EtOAc, and the combined

organic layers were washed with brine, dried over $\mathrm{Na}_{2} \mathrm{SO}_{4}$, and concentrated in vacuo to give a yellow oil.

The crude material was purified by column chromatography (hexane/diethyl ether 50:1) to afford an

inseparable E/Z mixture of (20S)-16 (470 mg, 86\%, E/Z 9:1) as a colorless oil. The following data were

collected as a 9:1 E/Z mixture; $[\alpha]_{D^{20}}^{20} 23.3\left(c 1.00, \mathrm{CHCl}_{3}\right) ; v_{\max }$ (neat) 2954, 2909, 2874, 1609, 1501, 1238, 
1042, $1018 \mathrm{~cm}^{-1}$; $\delta_{\mathrm{H}}\left(500 \mathrm{MHz}, \mathrm{CDCl}_{3}\right) 7.20(\mathrm{~d}, J=8.6 \mathrm{~Hz}, 1 \mathrm{H}), 6.70(\mathrm{dd}, J=8.6,2.6 \mathrm{~Hz}, 1 \mathrm{H}), 6.63(\mathrm{~d}, J=$ $2.0 \mathrm{~Hz}, 1 \mathrm{H}$ ), 5.52 (dt, $J=15.2,7.5 \mathrm{~Hz}, 0.9 \mathrm{H}), 5.50(\mathrm{dt}, J=10.9,3.7 \mathrm{~Hz}, 0.1 \mathrm{H}), 5.08$ (dd, $J=15.2,8.9 \mathrm{~Hz}$, 0.9H), 5.01 (dd, $J=10.3,10.3 \mathrm{~Hz}, 0.1 \mathrm{H}$ ), 3.77 (s, 3H), 2.95-2.75 (m, 2H), 2.34-2.02 (m, 5H), 1.97-1.84 (m, 2H), 1.50-1.28 (m, 7H), 1.27-1.18 (m, 1.6H), 1.18 (s, 2.7H), 1.17 (s, 2.7H), 1.15-1.05 (m, 2H), 0.95 (t, $J=$ $8.1 \mathrm{~Hz}, 9 \mathrm{H}), 0.78(\mathrm{~s}, 3 \mathrm{H}), 0.57(\mathrm{q}, J=8.0 \mathrm{~Hz}, 6 \mathrm{H}), 0.15$ (dd, $J=5.5,5.5 \mathrm{~Hz}, 1 \mathrm{H}) ; \delta_{\mathrm{c}}\left(125 \mathrm{MHz}, \mathrm{CDCl}_{3}\right)$ 157.4, 138.1, 134.3, 132.9, 126.3, 125.5, 113.8, 111.4, 73.5, 55.2, 53.5, 48.4, 43.9, 41.9, 39.4, 38.0, 33.4, 29.9, 29.7, 29.6, 27.8, 26.1, 24.7, 20.9, 19.2, 16.7, 7.1, 6.8 (Signals of the minor isomer were not observed); $\mathrm{m} / \mathrm{z}$ (EI) 508 (M), 493 (M-Me), 479 (M-Et), 450 (M-2Et). 376 (M-TESOH); HRMS (ESI): [M+Na] $]^{+}$, found 531.3631. $\mathrm{C}_{33} \mathrm{H}_{52} \mathrm{O}_{2} \mathrm{SiNa}$ requires 531.3629.

4.2.16. Synthesis

triethyl(((E)-5-((2'S,8S,9S,13S,14S,17S)-3-methoxy-13-methyl-6,7,8,9,11,12,13,14,15,16-decahydrospiro[cycl openta[a]phenanthrene-17,1'-cyclopropan]-2'-yl)-2-methylpent-4-en-2-yl)oxy)silane $\quad((20 R)-16) . \quad$ To $\quad$ a solution of 15 (747 mg, $1.87 \mathrm{mmol})$ in THF ( $5 \mathrm{~mL}$ ) was added a $1.0 \mathrm{M}$ solution of LiHMDS in toluene (1.9 $\mathrm{mL}, 1.9 \mathrm{mmol})$ at $-78{ }^{\circ} \mathrm{C}$ under argon. After being stirred for $30 \mathrm{~min}$, a solution of (20R)-13 (463 mg, 1.43 mmol) in THF $(10 \mathrm{~mL})$ was added, and stirred for $1 \mathrm{~h}$. The reaction mixture was diluted with EtOAc, and 
quenched with saturated aqueous $\mathrm{NH}_{4} \mathrm{Cl}$. The aqueous layer was extracted with EtOAc, and the combined organic layers were washed with brine, dried over $\mathrm{Na}_{2} \mathrm{SO}_{4}$, and concentrated in vacuo to give a yellow oil. The crude material was purified by column chromatography (hexane/diethyl ether 50:1) to afford an inseparable $E / Z$ mixture of (20R)-16 (667 mg, 92\%, E/Z 4:1) as a colorless oil. The following data were collected as a 4:1 E/Z mixture; $[\alpha]_{\mathrm{D}}^{20}+28.6$ (c 1.00, $\left.\mathrm{CHCl}_{3}\right) ; v_{\max }\left(\mathrm{CHCl}_{3}\right)$ 2932, 2913, 2874, 1609, 1501, 1458, 1234, 1153, 1038, $1015 \mathrm{~cm}^{-1} ; \delta_{\mathrm{H}}\left(500 \mathrm{MHz}, \mathrm{CDCl}_{3}\right) 7.20$ (d, $\left.J=8.6 \mathrm{~Hz}, 1 \mathrm{H}\right), 6.71$ (dd, $J=8.3,2.3 \mathrm{~Hz}$, 1H), $6.63(\mathrm{~d}, J=2.3 \mathrm{~Hz}, 1 \mathrm{H}), 5.54(\mathrm{dt}, J=15.2,7.2 \mathrm{~Hz}, 0.8 \mathrm{H}), 5.43(\mathrm{dt}, J=10.4,7.8 \mathrm{~Hz}, 0.2 \mathrm{H}), 5.29-5.18$ (m, 1H), 3.77 (s, 3H), 2.92-2.79 (m, 2H), 2.33-2.05 (m, 5H), 1.95-1.78 (m, 2H), 1.60-1.26 (m, 9H), 1.22 (s, 1.2H), 1.18 (s, 4.8H), 0.95 (t, $J=8.0 \mathrm{~Hz}, 9 \mathrm{H}), 0.88(\mathrm{~s}, 2.4 \mathrm{H}), 0.87$ (s, 0.6H), 0.83-0.78 (m, 1H), 0.57 (q, $J=$ 8.0 Hz, 6H), 0.54-0.51 (m, 1H); $\delta_{\mathrm{C}}\left(125 \mathrm{MHz}, \mathrm{CDCl}_{3}\right)$ 57.4, 138.0, 133.3, 132.9, 126.3, 125.5, 113.7, 111.4, 73.7, 55.2, 54.6, 48.6, 43.6, 41.7, 39.1, 37.9, 36.2, 35.7, 29.9, 29.8, 29.5, 27.7, 26.4, 25.7, 24.8, 20.3, 16.0, 7.1, 6.8 (Signals of the minor isomer were not observed); m/z (EI) 508 (M), 493 (M-Me), 479 (M-Et), 450 (M-2Et). 376 (M-TESOH); HRMS (ESI): [M+Na] $]^{+}$, found 531.3619. $\mathrm{C}_{33} \mathrm{H}_{52} \mathrm{O}_{2} \mathrm{SiNa}$ requires 531.3629.

4.2.17. Synthesis 
enanthrene-17,1'-cyclopropan]-2'-yl)-2-methylpentan-2-ol ((20S)-17). To a suspension of (20S)-16 (166 mg, $0.326 \mathrm{mmol}, \mathrm{E} / \mathrm{Z}$ 9:1) and dipotassium azodicarboxylate (317 $\mathrm{mg}, 1.63 \mathrm{mmol}$ ) in $\mathrm{CH}_{2} \mathrm{Cl}_{2}(1.0 \mathrm{~mL}$ ) was added a $1 \mathrm{M}$ solution of acetic acid in $\mathrm{CH}_{2} \mathrm{Cl}_{2}(3.3 \mathrm{~mL}, 3.3 \mathrm{mmol})$ dropwise at reflux. Additional dipotassium azodicarboxylate (317 mg, $1.63 \mathrm{mmol}$ ) and a $1.0 \mathrm{M}$ solution of acetic acid (3.3 mL, $3.3 \mathrm{mmol}$ ) were added after 3, 6, 22, 25, 28, 31, 34, $43 \mathrm{~h}$, respectively. The reaction mixture was stirred under reflux for a total $46 \mathrm{~h}$, and the resulting mixture was filtered through Celite. The eluent was concentrated under reduced pressure to give the spirocyclopropane (166 mg) as a pale yellow oil. To a solution of the spirocyclopropane (166 mg) in THF (2 mL) was added a 1.0 M solution of TBAF (1.7 mL, $1.7 \mathrm{mmol})$, and the solution was heated under reflux for $6 \mathrm{~h}$. After cooling to room temperature, the resulting mixture was diluted with EtOAc, and washed with water. The aqueous layer was extracted with EtOAc, dried over $\mathrm{Na}_{2} \mathrm{SO}_{4}$, and concentrated in vacuo to give a yellow oil. The crude material was purified by column chromatography (hexane/EtOAc 4:1) to give (20S)-17 (92.3 mg, 71\% for 2 steps) as a white solid; $\mathrm{Mp} 107-109{ }^{\circ} \mathrm{C}$; $[\alpha]_{\mathrm{D}}{ }^{20}+21.1\left(\mathrm{c} 1.00, \mathrm{CHCl}_{3}\right)$; $v_{\max }$ ( $\left.\mathrm{CHCl}_{3}\right) 3333$ (br), 2970, 2924, 2901, 1501, 1454, 1381, 1084, $1049 \mathrm{~cm}^{-1} ; \delta_{\mathrm{H}}\left(500 \mathrm{MHz}, \mathrm{CDCl}_{3}\right) 7.20$ (d, $J=$ 8.6 Hz, 1H), 6.70 (dd, $J=8.5,2.4 \mathrm{~Hz}, 1 \mathrm{H}), 6.63$ (d, $J=2.0 \mathrm{~Hz}, 1 \mathrm{H}), 3.77$ (s, 3H), 2.92-2.82 (m, 2H), 2.272.18 (m, 2H), 2.04-2.01 (m, 1H), 1.95-1.87 (m, 2H), 1.54-1.16 (m, 20H), 1.10 (ddd, $J=13.2,13.2,4.0 \mathrm{~Hz}$, 1H), 0.85 (dd, $J=8.9,4.3 \mathrm{~Hz}, 1 \mathrm{H}), 0.77$ (s, 3H), 0.58-0.52 (m, 1H), -0.26 (dd, $J=4.9,4.9 \mathrm{~Hz}, 1 \mathrm{H}) ; \delta_{\mathrm{C}}(125$ 
$\left.\mathrm{MHz}, \mathrm{CDCl}_{3}\right)$ 157.3, 138.0, 132.9, 126.2, 113.7, 111.3, 70.9, 55.1, 53.6, 43.9, 43.8, 41.3, 39.4, 36.0, 33.3, 31.6, 29.9, 29.2, 29.1, 29.0, 27.8, 26.1, 24.9, 24.6, 17.4, 17.1, 17.0; m/z (EI) 396 (M), 378 (M- $\left.\mathrm{H}_{2} \mathrm{O}\right), 363$ (M$\mathrm{H}_{2} \mathrm{O}-\mathrm{Me}$ ); HRMS (ESI): [M+H]+, found 397.3096. $\mathrm{C}_{27} \mathrm{H}_{41} \mathrm{O}_{2}$ requires 397.3101.

4.2.18.

Synthesis

5-((2'R,8S,9S,13S,14S,17S)-3-methoxy-13-methyl-6,7,8,9,11,12,13,14,15,16-decahydrospiro[cyclopenta[a]ph

enanthrene-17,1'-cyclopropan]-2'-yl)-2-methylpentan-2-ol ((20R)-17). To a suspension of (20R)-16 (483 mg,

$0.949 \mathrm{mmol}, \mathrm{E} / \mathrm{Z}$ 4:1) and dipotassium azodicarboxylate (922 mg, $4.75 \mathrm{mmol})$ in $\mathrm{CH}_{2} \mathrm{Cl}_{2}(5.0 \mathrm{~mL}$ ) was added

a $1.0 \mathrm{M}$ solution of acetic acid in $\mathrm{CH}_{2} \mathrm{Cl}_{2}(9.4 \mathrm{~mL}, 9.4 \mathrm{mmol})$ dropwise at reflux. Additional dipotassium

azodicarboxylate (922 mg, $4.75 \mathrm{mmol})$ and a $1.0 \mathrm{M}$ solution of acetic acid in $\mathrm{CH}_{2} \mathrm{Cl}_{2}(9.4 \mathrm{~mL}, 9.4 \mathrm{mmol})$

were added after $3,17,29,42,47,53,67,70,74,77 \mathrm{~h}$, respectively. The reaction mixture was stirred under

reflux for a total $89 \mathrm{~h}$, and the resulting mixture was filtered through Celite. The eluent was concentrated

under reduced pressure to give a pale yellow oil (402 mg, only about $50 \%$ conversion by ${ }^{1} \mathrm{H}$ NMR). Again, to

a suspension of the pale yellow oil (402 $\mathrm{mg}$ ) and dipotassium azodicarboxylate (768 mg, $3.95 \mathrm{mmol}$ ) in

$\mathrm{CH}_{2} \mathrm{Cl}_{2}(4.0 \mathrm{~mL})$ was added a $1.0 \mathrm{M}$ solution of acetic acid in $\mathrm{CH}_{2} \mathrm{Cl}_{2}(8.0 \mathrm{~mL}, 8.0 \mathrm{mmol})$ dropwise at reflux.

Additional dipotassium carboxylate $(770 \mathrm{mg}, 3.96 \mathrm{mmol})$ and a $1.0 \mathrm{M}$ solution of acetic acid in $\mathrm{CH}_{2} \mathrm{Cl}_{2}(8.0$ 
ml, 8.0 mmol) were added after 5, 9, 12, 32, 36, 49, 58 h, respectively. The reaction mixture was stirred under reflux for a total $72 \mathrm{~h}$, and the resulting white suspension was allowed to cool to room temperature, and diluted with water. The aqueous layer was extracted with $\mathrm{CHCl}_{3}$, and washed with brine, dried over $\mathrm{Na}_{2} \mathrm{SO}_{4}$. Concentration under reduced pressure gave a pale yellow oil (390 mg). To a solution of the pale yellow oil (390 mg, $0.763 \mathrm{mmol})$ in THF (5.0 mL) was added a 1.0 M solution of TBAF in THF (2.3 mL, $2.3 \mathrm{mmol})$. After being refluxed for $13 \mathrm{~h}$, the resulting mixture was allowed to cool to room temperature, and diluted with water. The aqueous layer was extracted with EtOAc twice. The combined organic layers were washed with brine, dried over $\mathrm{Na}_{2} \mathrm{SO}_{4}$, concentrated under reduced pressure to give a yellow oil. The residue was purified by column chromatography (hexane/EtOAc 5:1) to afford (20R)-17 (284 mg, 75\% for 2 steps) as a white solid. Mp 95-96 ${ }^{\circ} \mathrm{C} ;[\alpha]_{\mathrm{D}}^{20}+13.0$ (c 1.00, $\left.\mathrm{CHCl}_{3}\right)$; $v_{\max }\left(\mathrm{CHCl}_{3}\right) 3333$ (br), 2970, 2924, 2901, 1501, 1454, 1381, 1084, $1049 \mathrm{~cm}^{-1}$; $\delta_{\mathrm{H}}\left(500 \mathrm{MHz}, \mathrm{CDCl}_{3}\right) 7.19$ (d, $\left.J=8.6 \mathrm{~Hz}, 1 \mathrm{H}\right), 6.71$ (dd, $\left.J=8.6,2.6 \mathrm{~Hz}, 1 \mathrm{H}\right)$, 6.63 (d, $J=2.6 \mathrm{~Hz}, 1 \mathrm{H}), 3.77$ (s, 3H), 2.92-2.80 (m, 2H), 2.29-2.16 (m, 2H), 2.04-1.98 (m, 1H), 1.94-1.89 (m, 1H), 1.84-1.73 (m, 2H), 1.56-1.24 (m, 13H), 1.22 (s, 6H), 1.13-1.05 (m, 1H), 0.91 (s, 3H), 0.72 (m, 1H), 0.42 (dd, $J=4.9,4.9, \mathrm{~Hz}, 1 \mathrm{H}), 0.27$ (dd, $J=8.6,4.0 \mathrm{~Hz}, 1 \mathrm{H}) ; \delta_{\mathrm{C}}\left(125 \mathrm{MHz}, \mathrm{CDCl}_{3}\right) 157.4,138.1,132.9$, 126.2, 113.7, 111.4, 71.1, 55.2, 54.7, 43.9, 43.7, 41.6, 39.1, 36.7, 36.2, 35.6, 29.92, 29.90, 29.3, 29.2, 27.7, 26.5, 25.4, 24.8, 22.6, 18.4, 16.2; m/z (EI) 396 (M), $378\left(\mathrm{M}-\mathrm{H}_{2} \mathrm{O}\right), 363\left(\mathrm{M}-\mathrm{H}_{2} \mathrm{O}-\mathrm{Me}\right), 348\left(\mathrm{M}-\mathrm{H}_{2} \mathrm{O}-2 \mathrm{Me}\right)$; 
HRMS (ESI): $[\mathrm{M}+\mathrm{H}]^{+}$, found 397.3115. $\mathrm{C}_{27} \mathrm{H}_{41} \mathrm{O}_{2}$ requires 397.3101.

4.2.19.

Synthesis

(2'S,8S,9S,13S,14S,17S)-2'-(4-hydroxy-4-methylpentyl)-13-methyl-6,7,8,9,11,12,13,14,15,16-decahydrospiro[

cyclopenta[a]phenanthrene-17,1'-cyclopropan]-3-ol ((20S)-18). To a solution of diphenylphosphine (0.12

$\mathrm{mL}, 0.69 \mathrm{mmol})$ in THF $(1.0 \mathrm{~mL})$ were added a $2.5 \mathrm{M}$ solution of $n$-BuLi in hexane $(0.27 \mathrm{~mL}, 0.68 \mathrm{mmol})$

and a solution of (20S)-17 (52.0 mg, $0.132 \mathrm{mmol})$ in THF (1.0 mL). After the red solution was heated under

reflux for $25 \mathrm{~h}$, the resulting mixture was allowed to cool to room temperature. The mixture was diluted with

EtOAc, washed with $10 \% \mathrm{HCl}$, saturated aqueous $\mathrm{NaHCO}_{3}$ and brine, and dried over $\mathrm{Na}_{2} \mathrm{SO}_{4}$. Concentration

under reduced pressure gave a pale yellow oil, which was purified by column chromatography

(hexane/EtOAc 7:3) to give (20S)-18 (38.2 mg, 76\%) as a white solid; Mp 80-83 ${ }^{\circ} \mathrm{C}$; $[\alpha]_{\mathrm{D}}^{20}+9.85(c 0.50$,

$\mathrm{CHCl}_{3}$ ); $v_{\max }(\mathrm{KBr}) 3717$ (br), 3292 (br), 2968, 2932, 2864, 1611, 1585, 1501, 1452, 1375, 1287, $1240 \mathrm{~cm}^{-1}$;

$\delta_{\mathrm{H}}\left(500 \mathrm{MHz}, \mathrm{CDCl}_{3}\right) 7.15(\mathrm{~d}, J=8.6 \mathrm{~Hz}, 1 \mathrm{H}), 6.62(\mathrm{dd}, J=8.3,2.6 \mathrm{~Hz}, 1 \mathrm{H}), 6.56(\mathrm{~d}, J=2.3 \mathrm{~Hz}, 1 \mathrm{H}), 4.71$

(br s, $1 \mathrm{H}), 2.90-2.77$ (m, 2H), 2.30-2.13 (m, 2H), 2.09-1.99 (m, 1H), 1.97-1.83 (m, 2H), 1.55-1.21 (m,

19H), 1.16 (ddd, $J=12.3,2.9,2.9 \mathrm{~Hz}, 1 \mathrm{H}$ ), 1.08 (ddd, $J=12.6,12.6,4.0 \mathrm{~Hz}, 1 \mathrm{H}$ ), 0.85 (dd, $J=9.2,4.3 \mathrm{~Hz}$,

1H), 0.76 (s, 3H), 0.58-0.51 (m, 1H), -0.26 (dd, $J=4.9,4.9 \mathrm{~Hz}, 1 \mathrm{H})$; $\delta_{\mathrm{C}}\left(125 \mathrm{MHz}, \mathrm{CDCl}_{3}\right) 153.4,138.4$, 
132.9, 126.4, 115.2, 112.6, 71.5, 53.7, 44.0, 43.8, 41.4, 39.5, 36.0, 33.3, 31.6, 29.7, 29.2, 29.1, 29.0, 27.8, 26.2, 24.9, 24.6, 17.5, 17.11, 17.07; m/z (EI) 382 (M), 364 (M- $\left.\mathrm{H}_{2} \mathrm{O}\right), 349$ (M- $\left.\mathrm{H}_{2} \mathrm{O}-\mathrm{Me}\right)$; HRMS (ESI):

$[\mathrm{M}+\mathrm{H}]^{+}$, found 383.2931. $\mathrm{C}_{26} \mathrm{H}_{39} \mathrm{O}_{2}$ requires 383.2945.

4.2.20.

Synthesis

of

(2'R,8S,9S,13S,14S,17S)-2'-(4-hydroxy-4-methylpentyl)-13-methyl-6,7,8,9,11,12,13,14,15,16-decahydrospiro[

cyclopenta[a]phenanthrene-17,1'-cyclopropan]-3-ol ((20R)-18). To a solution of diphenylphosphine (0.33 ml, $1.90 \mathrm{mmol})$ in THF $(1 \mathrm{ml})$ were added a $2.5 \mathrm{M}$ solution of $n$-BuLi in hexane $(0.76 \mathrm{ml}, 1.90 \mathrm{mmol})$ and a solution of (20R)-17 (151 mg, $0.381 \mathrm{mmol})$ in THF (3 ml). After the red solution was heated under reflux for $21 \mathrm{~h}$, the resulting mixture was allowed to cool to room temperature. The mixture was diluted with EtOAc, washed with $10 \% \mathrm{HCl}$, saturated aqueous $\mathrm{NaHCO}_{3}$ and brine, and dried over $\mathrm{Na}_{2} \mathrm{SO}_{4}$. Concentration under reduced pressure gave a pale yellow oil, which was purified by column chromatography (hexane/EtOAc 4:1 to 7:3) to give (20R)-18 (133 mg, 91\%) as a white solid; Mp $192-193{ }^{\circ} \mathrm{C} ;[\alpha]_{\mathrm{D}}{ }^{20}+14.1$ (c 1.00, THF); $v_{\max }$ (KBr) 3319 (br), 2968, 2857, 2816, 1614, 1585, 1504, 1440, 1375, 1283, 1213, 1148, $895 \mathrm{~cm}^{-1}$; $\delta_{\mathrm{H}}(500 \mathrm{MHz}$, $\left.\mathrm{CDCl}_{3}\right) 7.14(\mathrm{~d}, J=8.3 \mathrm{~Hz}, 1 \mathrm{H}), 6.62(\mathrm{dd}, J=8.3,2.0 \mathrm{~Hz}, 1 \mathrm{H}), 6.56$ (d, $\left.J=2.3 \mathrm{~Hz}, 1 \mathrm{H}\right), 2.88-2.78(\mathrm{~m}, 2 \mathrm{H})$, 2.26-2.15 (m, 2H), 2.05-1.98 (m, 1H), 1.93-1.87 (m, 1H), 1.84-1.73 (m, 2H), 1.56-1.29 (m, 14H), 1.23 (s, 
6H), 1.10-1.05 (m, 1H), 0.91 (s, 3H), 0.72 (m, 1H), 0.42 (dd, $J=4.3,4.3, \mathrm{~Hz}, 1 \mathrm{H}), 0.27$ (dd, $J=8.6,4.0 \mathrm{~Hz}$, $1 \mathrm{H}) ; \delta_{\mathrm{C}}\left(125 \mathrm{MHz}\right.$, acetone- $\left.d_{6}\right) 156.5,139.0,132.7,127.5,116.5,114.1,70.7,56.2,45.4,45.2,43.0,40.9$, 38.2, 37.5, 37.0, 31.5, 31.0, 30.4, 29.1, 27.9, 26.8, 26.0, 24.1, 19.5, 17.3; m/z (EI) 382 (M), $364\left(\mathrm{M}-\mathrm{H}_{2} \mathrm{O}\right)$, 349 (M- $\left.\mathrm{H}_{2} \mathrm{O}-\mathrm{Me}\right)$; HRMS (ESI): [M+Na] ${ }^{+}$, found 405.2772. $\mathrm{C}_{26} \mathrm{H}_{38} \mathrm{O}_{2} \mathrm{Na}$ requires 405.2764.

4.2.21. Synthesis

tert-butyl(((6bS,8aS,8bR,9S,10aS,11aS,11bR)-4-methoxy-8a-methyl-9-((E)-4-methyl-4-((triethylsilyl)oxy)pent -1-en-1-yl)-1,2,6b,7,8,8a,9,10,10a,11,11a,11b-dodecahydro-8bH-cyclobuta[3,4]cyclopenta[1,2-a]phenanthre n-8b-yl)oxy)dimethylsilane (trans-19). To a solution of oxalyl chloride (0.38 mL, $4.4 \mathrm{mmol})$ in $\mathrm{CH}_{2} \mathrm{Cl}_{2}(5.0$ $\mathrm{mL})$ was dropwise added a solution of DMSO $(0.47 \mathrm{~mL}, 6.6 \mathrm{mmol})$ in $\mathrm{CH}_{2} \mathrm{Cl}_{2}(6.0 \mathrm{~mL})$ at $-78{ }^{\circ} \mathrm{C}$. After being stirred for $5 \mathrm{~min}$, trans-7 $(1.00 \mathrm{~g}, 2.19 \mathrm{mmol})$ in $\mathrm{CH}_{2} \mathrm{Cl}_{2}(11 \mathrm{~mL})$ was dropwise added. After being stirred for $40 \mathrm{~min}$ at $-78{ }^{\circ} \mathrm{C}$, triethylamine $(1.8 \mathrm{~mL}, 13 \mathrm{mmol})$ was added. After being stirred for $20 \mathrm{~min}$, the resulting mixture was allowed to warm to ambient temperature, diluted with $\mathrm{CHCl}_{3}$, and quenched with saturated aqueous $\mathrm{NH}_{4} \mathrm{Cl}$. The aqueous layer was extracted with $\mathrm{CHCl}_{3}$, and the combined organic layers were washed with brine, dried over $\mathrm{Na}_{2} \mathrm{SO}_{4}$, and concentrated under reduced pressure. The residue was dissolved in $\mathrm{CHCl}_{3}$ and passed through a short pad of silica gel with hexane/diethyl ether (10:1) to obtain a 
crude aldehyde (936 mg) as a white solid, which was used directly in the next step without further purification. To a solution of $15(1.07 \mathrm{~g}, 2.69 \mathrm{mmol})$ in THF $(7 \mathrm{~mL})$ was added a $1.0 \mathrm{M}$ solution of LiHMDS in toluene $(2.7 \mathrm{~mL}, 2.7 \mathrm{mmol})$ at $-78{ }^{\circ} \mathrm{C}$ under argon. After being stirred for $30 \mathrm{~min}$, a solution of the above crude aldehyde (936 mg, $2.06 \mathrm{mmol}$ ) in THF (13 mL) was added, and stirred for $1 \mathrm{~h}$. The reaction mixture was diluted with EtOAc, and quenched with saturated aqueous $\mathrm{NH}_{4} \mathrm{Cl}$. The aqueous layer was extracted with EtOAc, and the combined organic layers were washed with brine, dried over $\mathrm{Na}_{2} \mathrm{SO}_{4}$, and concentrated in vacuo to give a yellow solid. The crude material was purified by column chromatography (hexane/diethyl ether 50:1) to afford an inseparable $E / Z$ mixture of trans-19 (1.18 g, 84\% for 2 steps, $E / Z$ 3:2) as a colorless oil. The following data were collected as a 3:2 $\mathrm{E} / \mathrm{Z}$ mixture; $[\alpha]_{\mathrm{D}}^{20}+11.5$ (c 1.00, $\left.\mathrm{CHCl}_{3}\right) ; v_{\max }\left(\mathrm{CHCl}_{3}\right) 2955$, 2928, 1501, 1458, 1254, 1238, $1045 \mathrm{~cm}^{-1}$; $\delta_{\mathrm{H}}\left(500 \mathrm{MHz}, \mathrm{CDCl}_{3}\right) 7.22$ (d, $\left.J=8.6 \mathrm{~Hz}, 0.4 \mathrm{H}\right), 7.21$ (d, $J=8.6$ Hz, 0.6H), 6.71 (dd, $J=8.6,2.9 \mathrm{~Hz}, 1 \mathrm{H}), 6.63(\mathrm{~d}, J=2.9 \mathrm{~Hz}, 1 \mathrm{H}$ ), 5.86-5.77 (m, 1H), 5.49 (ddt, $J=10.9,7.5$, $0.9 \mathrm{~Hz}, 0.4 \mathrm{H}), 5.43$ (ddt, $J=15.5,7.2,1.4 \mathrm{~Hz}, 0.6 \mathrm{H}), 3.77$ (s, 3H), 3.44-3.36 (m, 0.4H), 3.26-3.18 (m, 0.6H), 2.93-2.81 (m, 2H), 2.60-2.54 (m, 0.4H), 2.51-2.42 (m, 1H), 2.38-2.28 (m, 1H), 2.27-2.13 (m, 4H), 2.06 (ddd, $J=3.8,3.8,3.2 \mathrm{~Hz}, 0.6 \mathrm{H}), 1.97-1.90$ (m, 1H), 1.89-1.78 (m, 1H), 1.60-1.33 (m, 6H), 1.20 (s, 1.2H), 1.18 (s, 1.2H), 1.17 (s, 3.6H), 1.08-0.89 (m, 19H), 0.70 (s, 1.8H), 0.69 (s, 1.2H), 0.57 (q, J = 8.1 Hz, 2.4H), 0.56 (q, $J=8.0 \mathrm{~Hz}, 3.6 \mathrm{H}), 0.27$ (s, 1.2H), 0.21 (s, 1.8H), 0.133 (s, 1.8H), 0.127 (s, 1.2H); $\delta_{\mathrm{c}}(125 \mathrm{MHz}$, 
$\left.\mathrm{CDCl}_{3}\right) 157.4,157.3,138.1,138.0,133.02,133.0,132.7,132.6,127.0,126.5,126.4,125.6,113.7,111.41$, 111.38, 92.0, 91.7, 73.6, 73.4, 55.2, 50.5, 48.47, 48.45, 48.3, 47.1, 47.0, 45.8, 44.0, 43.8, 43.3, 41.5, 41.0, 38.87, 38.85, 33.7, 33.6, 31.2, 30.0, 29.94, 29.91, 29.85, 29.7, 28.7, 27.89, 27.85, 26.42, 26.37, 26.0, 25.9, 25.8, 18.3, 17.01, 16.95, 7.1, 6.8, -2.4, -2.7, -3.1, -3.3 (some signals missing); m/z (EI) 638 (M), 623 (MMe), 609 (M-Et), 581 (M-tBu), 506 (M-TESOH); HRMS (ESI): [M+Na] $]^{+}$, found 661.4428. $\mathrm{C}_{39} \mathrm{H}_{66} \mathrm{O}_{3} \mathrm{Si}_{2} \mathrm{Na}$ requires 661.4442 .

4.2.22. Synthesis tert-butyl(((6bS,8aS,8bR,9R,10aS,11aS,11bR)-4-methoxy-8a-methyl-9-((E)-4-methyl-4-((triethylsilyl)oxy)pent -1-en-1-yl)-1,2,6b,7,8,8a,9,10,10a,11,11a,11b-dodecahydro-8bH-cyclobuta[3,4]cyclopenta[1,2-a]phenanthre n-8b-yl)oxy)dimethylsilane (cis-19). To a solution of oxalyl chloride (0.49 mL, $5.7 \mathrm{mmol})$ in $\mathrm{CH}_{2} \mathrm{Cl}_{2}(7.0 \mathrm{~mL})$ was dropwise added a solution of DMSO $(0.61 \mathrm{~mL}, 8.6 \mathrm{mmol})$ in $\mathrm{CH}_{2} \mathrm{Cl}_{2}(6.0 \mathrm{~mL})$ at $-78{ }^{\circ} \mathrm{C}$. After being stirred for $5 \mathrm{~min}$, a solution of cis-7 (1.30 g, $2.85 \mathrm{mmol})$ in $\mathrm{CH}_{2} \mathrm{Cl}_{2}(15 \mathrm{~mL})$ was dropwise added. After being stirred for $40 \mathrm{~min}$ at $-78{ }^{\circ} \mathrm{C}$, triethylamine $(2.4 \mathrm{~mL}, 17 \mathrm{mmol})$ was added. After being stirred for $20 \mathrm{~min}$, the resulting mixture was allowed to warm to ambient temperature, diluted with $\mathrm{CHCl}_{3}$, and quenched with saturated aqueous $\mathrm{NH}_{4} \mathrm{Cl}$. The aqueous layer was extracted with $\mathrm{CHCl}_{3}$, and the combined organic layers 
were washed with brine, dried over $\mathrm{Na}_{2} \mathrm{SO}_{4}$, and concentrated under reduced pressure. The residue was dissolved in $\mathrm{CHCl}_{3}$ and passed through a short pad of silica gel with hexane/EtOAc (20:1) to obtain a crude aldehyde (1.21 g) as a white solid, which was used directly in the next step without further purification. To a solution of 15 (1.39 g, $3.48 \mathrm{mmol})$ in THF (8.0 mL) was added a 1.0 M solution of LiHMDS in toluene (3.5 $\mathrm{mL}, 3.5 \mathrm{mmol}$ ) at $-78{ }^{\circ} \mathrm{C}$ under argon. After being stirred for $30 \mathrm{~min}$, a solution of the above crude aldehyde (1.21 g, $2.66 \mathrm{mmol})$ in THF (19 mL) was added, and stirred for $1 \mathrm{~h}$. The reaction mixture was diluted with EtOAc, and quenched with saturated aqueous $\mathrm{NH}_{4} \mathrm{Cl}$. The aqueous layer was extracted with EtOAc, and the combined organic layers were washed with brine, dried over $\mathrm{Na}_{2} \mathrm{SO}_{4}$, and concentrated in vacuo to give a yellow oil. The crude material was purified by column chromatography (hexane/EtOAc 50:1) to afford an inseparable E/Z mixture of cis-19 (1.29 g, 71\% for 2 steps, E/Z 1:1) as a colorless oil. The following data were collected as a 1:1 E/Z mixture; $[\alpha]_{\mathrm{D}}^{20}+4.95$ (c 1.00, $\left.\mathrm{CHCl}_{3}\right) ; v_{\max }\left(\mathrm{CHCl}_{3}\right) 2951,2928,2878,2855$, 1501, 1462, 1234, 1153, 1099, 1045, $1018 \mathrm{~cm}^{-1}$; $\delta_{\mathrm{H}}\left(500 \mathrm{MHz}, \mathrm{CDCl}_{3}\right) 7.23$ (d, $\left.J=8.9 \mathrm{~Hz}, 1 \mathrm{H}\right), 6.72$ (dd, $J=$ 8.6, $2.3 \mathrm{~Hz}, 1 \mathrm{H}$ ), 6.64 (d, $J=2.3 \mathrm{~Hz}, 1 \mathrm{H}$ ), 5.74 (dt, $J=10.0,7.9 \mathrm{~Hz}, 0.5 \mathrm{H}), 5.43$ (dd, $J=15.5,6.1 \mathrm{~Hz}, 0.5 \mathrm{H}$ ), 5.45-5.32 (m, 1H), 3.78 (s, 3H), 3.27-3.21 (m, 0.5H), 3.11-3.04 (m, 0.5H), 2.96-2.82 (m, 2H), 2.60 (dd, $J=$ 7.5, 7.5 Hz, 0.5H), 2.54 (dd, $J=8.0,8.0 \mathrm{~Hz}, 0.5 \mathrm{H}), 2.35-2.05$ (m, 5H), 1.99-1.90 (m, 1H), 1.74-1.64 (m, 1H), 1.62-1.38 (m, 8H), 1.20 (s, 1.5H), 1.19 (s, 1.5H), 1.18 (s, 1.5H), 1.16 (s, 1.5H), 0.95 (t, $J=8.0 \mathrm{~Hz}, 9 \mathrm{H})$, 
0.92 (s, 4.5H), 0.90 (s, 4.5H), 0.70 (s, 1.5H), 0.69 (s, 1.5H), 0.574 (q, $J=7.7 \mathrm{~Hz}, 3 \mathrm{H}), 0.569$ (q, $J=8.1 \mathrm{~Hz}$, 3H), 0.26 (s, 1.5H), 0.22 (s, 1.5H), 0.10 (s, 1.5H), 0.08 (s, 1.5H); $\delta_{\mathrm{C}}\left(125 \mathrm{MHz}, \mathrm{CDCl}_{3}\right)$ 157.4, 137.9,134.1, 132.90, 132.87, 132.8, 126.3, 126.1, 125.3, 113.8, 111.4, 90.7, 90.5, 73.6, 73.5, 55.1, 48.4, 48.34, 48.32, 47.0, 46.9, 44.31, 44.27, 43.4, 40.8, 40.5, 40.3, 38.7, 38.6, 35.9, 32.1, 31.9, 30.4, 30.0, 29.9, 29.80, 29.76, 29.6, 29.5, 29.3, 28.3, 27.8, 26.14, 26.10, 18.64, 18.56, 14.6, 14.5, 7.11, 6.8, $-1.46,-1.51,-2.4$ ppm (some signals missing); m/z (EI) 638 (M), 609 (M-Et), 581 (M-tBu), 506 (M-TESOH); HRMS (ESI): [M+Na] ${ }^{+}$, found 661.4439. $\mathrm{C}_{39} \mathrm{H}_{66} \mathrm{O}_{3} \mathrm{Si}_{2} \mathrm{Na}$ requires 661.4442.

4.2.23.

Synthesis

5-((6bS,8aS,8bS,9R,10aS,11aS,11bR)-8b-((tert-butyldimethylsilyl)oxy)-4-methoxy-8a-methyl-2,6b,7,8,8a,8b,9 ,10,10a,11,11a,11b-dodecahydro-1H-cyclobuta[3,4] cyclopenta[1,2-a]phenanthren-9-yl)-2-methylpentan-2-ol

(trans-20). A mixture of trans-19 (1.18 g, 1.85 mmol, E/Z 3:2), 10\% Pd/C (200 mg) and EtOAc/MeOH (1:1, $18 \mathrm{~mL}$ ) was stirred under $\mathrm{H}_{2}(1 \mathrm{~atm})$ at room temperature for $10 \mathrm{~h}$. The reaction mixture was filtered through Celite, and concentrated under reduced pressure to give a pale yellow oil, which was purified by column chromatography (hexane/diethyl ether 3:2) to afford trans-20 (626 mg, 64\%) as a white solid; Mp 52-55 ${ }^{\circ} \mathrm{C}$; $[\alpha]_{\mathrm{D}}^{20}+9.64$ (c 1.00, $\left.\mathrm{CHCl}_{3}\right) ; v_{\max }\left(\mathrm{CHCl}_{3}\right) 3422$ (br), 2940, 2862, 1612, 1485, 1454, 1238, 1211, $1103 \mathrm{~cm}^{-1}$; 
$\delta_{\mathrm{H}}\left(500 \mathrm{MHz}, \mathrm{CDCl}_{3}\right) 7.22(\mathrm{~d}, J=8.6 \mathrm{~Hz}, 1 \mathrm{H}), 6.71(\mathrm{dd}, J=8.6,2.6 \mathrm{~Hz}, 1 \mathrm{H}), 6.67(\mathrm{~d}, J=2.3 \mathrm{~Hz}, 1 \mathrm{H}), 3.78$ (s, 3H), 2.90-2.82 (m, 2H), 2.59 (dddd, $J=12.6,12.6,8.0,5.5 \mathrm{~Hz}, 1 \mathrm{H}), 2.48-2.44$ (m, 1H), 2.36-2.22 (m, 3H), 1.95-1.88 (m, 2H), 1.84-1.72 (m, 2H), 1.68-1.64 (m, 2H), 1.56-1.38 (m, 7H), 1.36-1.28 (m, 1H), 1.21 (s, 6H), 1.16-1.09 (m, 1H), 0.89 (s, 9H), 0.82 (ddd, $J=12.9,8.6,4.9 \mathrm{~Hz}, 1 \mathrm{H}), 0.72$ (s, 3H), 0.18 (s, 3H), 0.11 (s, 3H); $\delta_{\mathrm{C}}\left(125 \mathrm{MHz}, \mathrm{CDCl}_{3}\right)$ 157.3, 138.1, 132.9, 126.4, 113.7, 111.4, 90.4, 71.0, 55.2, 48.8, 48.6, 47.5, 44.0, 43.9, 41.5, 39.0, 33.3, 31.9, 31.4, 30.0, 29.3, 29.2, 27.9, 27.4, 26.5, 25.9, 23.1, 18.3, 17.5, -2.7, -3.0; m/z (EI) 526 (M), 511 (M-Me), 411 (M-TBS); HRMS (ESI): $[\mathrm{M}+\mathrm{Na}]^{+}$, found 549.3752. $\mathrm{C}_{33} \mathrm{H}_{54} \mathrm{O}_{3} \mathrm{SiNa}$ requires 549.3734 .

4.2.24.

Synthesis

5-((6bS,8aS,8bS,9S,10aS,11aS,11bR)-8b-((tert-butyldimethylsilyl)oxy)-4-methoxy-8a-methyl-2,6b,7,8,8a,8b,9 ,10,10a,11,11a,11b-dodecahydro-1H-cyclobuta[3,4] cyclopenta[1,2-a]phenanthren-9-yl)-2-methylpentan-2-ol (cis-20). A mixture of cis-19 (1.29 g, 2.02 mmol, E/Z 1:1), 10\% Pd/C (215 mg) and EtOAc/MeOH (1:1, 20 $\mathrm{mL}$ ) was stirred under $\mathrm{H}_{2}(1 \mathrm{~atm})$ at room temperature for $10 \mathrm{~h}$. The reaction mixture was filtered through Celite, and concentrated under reduced pressure to give a white gum, which was purified by column chromatography (hexane/diethyl ether 3:2) to afford cis-20 (847 mg, 80\%) as a white solid; Mp 63-66 ${ }^{\circ} \mathrm{C}$; 
$[\alpha]_{D}^{20}+19.0$ (c 1.00, $\left.\mathrm{CHCl}_{3}\right) ; v_{\max }$ (neat) 3325 (br), 2970, 2928, 1612, 1500, 1254, 1092, $1045 \mathrm{~cm}^{-1} ; \delta_{\mathrm{H}}(500$

MHz, $\left.\mathrm{CDCl}_{3}\right) 7.22$ (d, $\left.J=8.6 \mathrm{~Hz}, 1 \mathrm{H}\right), 6.72$ (dd, $\left.J=8.6,2.6 \mathrm{~Hz}, 1 \mathrm{H}\right), 6.64$ (d, $\left.J=2.6 \mathrm{~Hz}, 1 \mathrm{H}\right), 3.78$ (s, 3H),

2.89-2.83 (m, 2H), 2.54 (dd, $J=7.5,7.5 \mathrm{~Hz}, 1 \mathrm{H}), 2.38-2.30$ (m, 2H), 2.23 (dd, $J=9.9,9.9 \mathrm{~Hz}, 1 \mathrm{H}$ ), 1.93-

1.83 (m, 2H), 1.68-1.21 (m, 15H), 1.20 (s, 6H), 0.91 (s, 9H), 0.69 (s, 3H), 0.24 (s, 3H), 0.11 (s, 3H); $\delta_{\mathrm{C}}(125$

$\left.\mathrm{MHz}, \mathrm{CDCl}_{3}\right)$ 157.4, 138.0, 132.9, 126.4, 113.8, 111.4, 90.2, 71.1, 55.2, 48.3, 46.6, 44.4, 44.3, 40.0, 38.6,

38.1, 32.0, 31.9, 29.9, 29.7, 29.5, 29.2, 29.0, 27.8, 26.2, 23.0, 18.6, 14.6, -1.2, -2.3; m/z (EI) 526 (M), 511

(M-Me), 411 (M-TBS); HRMS (ESI): [M+Na] ${ }^{+}$, found 549.3737. $\mathrm{C}_{33} \mathrm{H}_{54} \mathrm{O}_{3} \mathrm{SiNa}$ requires 549.3734.

4.2.25.

Synthesis

of

(6bS,8aS,8bS,9R,10aS,11aS,11bR)-8b-((tert-butyldimethylsilyl)oxy)-9-(4-hydroxy-4-methylpentyl)-8a-methyl-

2,6b,7,8,8a,8b,9,10,10a,11,11a,11b-dodecahydro-1H-cyclobuta[3,4]cyclopenta[1,2-a]phenanthren-4-ol

(trans-21). To a solution of diphenylphosphine $(0.53 \mathrm{ml}, 3.1 \mathrm{mmol})$ in THF $(4.0 \mathrm{~mL})$ were added a $2.5 \mathrm{M}$

solution of $n$-BuLi in hexane (1.2 mL, $3.0 \mathrm{mmol})$ and a solution of trans-20 (533 mg, $1.01 \mathrm{mmol})$ in THF

(6.0 mL). The red solution was heated under reflux for $27 \mathrm{~h}$, and the diphenyl phosphine ( $0.35 \mathrm{~mL}, 2.0$

mmol) and a $2.5 \mathrm{M}$ solution of $n$-BuLi in hexane $(0.81 \mathrm{~mL}, 2.0 \mathrm{mmol})$ were added again to the mixture. After

being stirred for additional $19 \mathrm{~h}$ under reflux, the resulting mixture was allowed to cool to room temperature. 
The mixture was diluted with EtOAc, washed with $10 \% \mathrm{HCl}$, saturated aqueous $\mathrm{NaHCO}_{3}$ and brine, and dried over $\mathrm{Na}_{2} \mathrm{SO}_{4}$. Concentration under reduced pressure gave a pale yellow oil, which was purified by column chromatography (hexane/EtOAc 3:1) to give trans-21 (458 mg, 88\%) as a white solid. Analytical sample was obtained by recrystallization from EtOAc as a white powder; Found 74.70; $\mathrm{H}, 10.29 . \mathrm{C}_{32} \mathrm{H}_{52} \mathrm{O}_{3} \mathrm{Si}$ requires C, 74.94; $\mathrm{H}, 10.22 \%$; $\mathrm{Mp} 178-179{ }^{\circ} \mathrm{C} ;[\alpha]_{\mathrm{D}}^{20}+5.35$ (c 1.00, $\mathrm{CHCl}_{3}$ ); $v_{\max }$ (neat) 3329 (br), 2974, 2928, 2893, 1454, 1088, $1049 \mathrm{~cm}^{-1} ; \delta_{\mathrm{H}}\left(500 \mathrm{MHz}, \mathrm{CDCl}_{3}\right) 7.17$ (d, $\left.J=8.3 \mathrm{~Hz}, 1 \mathrm{H}\right), 6.63$ (dd, $J=8.5,2.7 \mathrm{~Hz}$, 1H), 6.57 (d, $J=2.6 \mathrm{~Hz}, 1 \mathrm{H}), 2.88-2.79$ (m, 2H), 2.62-2.54 (m, 1H), 2.48-2.44 (m, 1H), 2.32 (ddd, $J=12.0$, 12.0, $9.5 \mathrm{~Hz}, 1 \mathrm{H}), 2.26-2.20$ (m, 2H), 1.94-1.88 (m, 2H), 1.84-1.72 (m, 2H), 1.67-1.60 (m, 2H), 1.57-1.25 (m, 9H), 1.21 (s, 6H), 1.16-1.09 (m, 1H), 0.89 (s, 9H), 0.82 (ddd, $J=12.2,8.5,5.2 \mathrm{~Hz}, 1 \mathrm{H}), 0.72$ (s, 3H), 0.18 (s, 3H), 0.11 (s, 3H); $\delta_{\mathrm{C}}\left(125 \mathrm{MHz}, \mathrm{CDCl}_{3}\right)$ 153.4, 138.3, 132.7, 126.6, 115.2, 112.6, 90.4, 71.4, 48.8, 48.6, 47.5, 43.9, 41.5, 38.9, 33.3, 31.9, 31.4, 29.8, 29.2, 29.1, 27.8, 27.3, 26.5, 25.9, 23.1, 18.3, 17.5, 18.3, 17.5, -2.7, -3.0; m/z (EI) 512 (M), 453 (M-2Me-OH), 397 (M-TBS).

4.2.26.

Synthesis

(6bS,8aS,8bS,9S,10aS,11aS,11bR)-8b-((tert-butyldimethylsilyl)oxy)-9-(4-hydroxy-4-methylpentyl)-8a-methyl2,6b,7,8,8a,8b,9,10,10a,11,11a,11b-dodecahydro-1H-cyclobuta[3,4]cyclopenta[1,2-a]phenanthren-4-ol 
(cis-21). To a solution of diphenylphosphine $(0.99 \mathrm{~mL}, 5.7 \mathrm{mmol})$ in THF $(4.0 \mathrm{~mL})$ were added a $2.5 \mathrm{M}$ solution of $n$-BuLi in hexane (2.3 mL, $5.8 \mathrm{mmol})$ and a solution of cis-20 (603 mg, $1.14 \mathrm{mmol})$ in THF (7.0 $\mathrm{mL}$ ). After the red solution was heated under reflux for $20 \mathrm{~h}$, the resulting mixture was allowed to cool to room temperature. The mixture was diluted with EtOAc, washed with $10 \% \mathrm{HCl}$, saturated aqueous $\mathrm{NaHCO}_{3}$ and brine, and dried over $\mathrm{Na}_{2} \mathrm{SO}_{4}$. Concentration under reduced pressure gave a pale yellow oil, which was purified by column chromatography (hexane/EtOAc 3:1) to give cis-21 (455 mg, 78\%) as a white solid. Analytical sample was obtained by recrystallization from EtOAc as a white powder; C, 74.79; H, 10.44. $\mathrm{C}_{32} \mathrm{H}_{52} \mathrm{O}_{3} \mathrm{Si}$ requires C, 74.94; H, 10.22\%; Mp 205-206 ${ }^{\circ} \mathrm{C}$; $[\alpha]_{\mathrm{D}}^{20}+21.5$ (c 1.00, THF); $v_{\text {max }}$ (neat) 3314 (br), 2974, 2882, 1088, $1045 \mathrm{~cm}^{-1}$; $\delta_{\mathrm{H}}\left(500 \mathrm{MHz}, \mathrm{CDCl}_{3}\right) 7.17$ (d, $\left.J=8.3 \mathrm{~Hz}, 1 \mathrm{H}\right), 6.64$ (dd, $\left.J=8.2,2.4 \mathrm{~Hz}, 1 \mathrm{H}\right)$, 6.57 (d, $J=2.3 \mathrm{~Hz}, 1 \mathrm{H}), 4.56$ (s, 1H), 2.85-2.82 (m, 2H), 2.54 (ddd, $J=8.4,8.4,1.6 \mathrm{~Hz}, 1 \mathrm{H}), 2.35-2.28$ (m, 2H), 2.23-2.19 (m, 1H), 1.92-1.83 (m, 2H), 1.67-1.24 (m, 16H), 1.21 (s, 3H), 1.20 (s, 3H), 0.91 (s, 9H), 0.69 (s, 3H), 0.24 (s, 3H), 0.10 (s, 3H); $\delta_{\mathrm{C}}\left(125 \mathrm{MHz}, \mathrm{THF}-\mathrm{d}_{8}\right)$ 156.4, 138.1, 131.6, 126.7, 115.9, 113.6, 91.4, 69.7, 49.3, 47.6, 45.5, 45.4, 41.0, 40.0, 39.2, 33.3, 32.7, 30.7, 30.6, 30.1, 29.4, 28.9, 27.2, 26.7, 23.8, 19.4, 15.3, 1.0, $-2.0 ; \mathrm{m} / \mathrm{z}(\mathrm{EI}) 512(\mathrm{M})$. 
(6bS,8aS,8bS,9R,10aS,11aS,11bR)-9-(4-hydroxy-4-methylpentyl)-8a-methyl-1,2,6b,7,8,8a,9,10,10a,11,11a,11

b-dodecahydro-8bH-cyclobuta[3,4]cyclopenta[1,2-a]phenanthrene-4,8b-diol (trans-22). To a solution of trans-21 $(120 \mathrm{mg}, 0.234 \mathrm{mmol})$ in THF $(1.0 \mathrm{~mL})$ was added a $1.0 \mathrm{M}$ solution of TBAF in THF $(1.2 \mathrm{~mL}, 1.2$ mmol), and the solution was heated under reflux for $24 \mathrm{~h}$. The resulting mixture was allowed to cool to room temperature, and purified by column chromatography (hexane/EtOAc 2:3) to give trans-22 (71 mg, 76\%) as a white solid; Mp $197-198{ }^{\circ} \mathrm{C}$; $[\alpha]_{\mathrm{D}}{ }^{20}+12.8$ (c 1.00, THF); $v_{\max }$ (THF) 3348 (br), 1454, 1361, 1288, 1222, $1080 \mathrm{~cm}^{-1} ; \delta_{\mathrm{H}}\left(500 \mathrm{MHz}, \mathrm{MeOH}-d_{4}\right) 7.09(\mathrm{~d}, J=8.6 \mathrm{~Hz}, 1 \mathrm{H}), 6.54$ (dd, $\left.J=8.3,2.6 \mathrm{~Hz}, 1 \mathrm{H}\right), 6.48(\mathrm{~d}, J=1.7$ Hz, 1H), 2.81-2.76 (m, 2H), 2.62-2.55 (m, 1H), 2.42-2.23 (m, 3H), 2.21 (ddd, $J=11.1,11.1,3.0 \mathrm{~Hz}, 1 \mathrm{H}$ ), 2.02-1.91 (m, 3H), 1.80-1.65 (m, 3H), 1.56-1.33 (m, 9H), 1.161 (s, 3H), 1.158 (s, 3H), 0.86 (m, 1H), 0.80 (s, 3H); $\delta_{\mathrm{C}}\left(125 \mathrm{MHz}, \mathrm{MeOH}-d_{4}\right) 155.9,138.8,132.6,127.3,116.0,113.7,89.2,71.4,50.0,49.6,48.9,45.3$, 44.8, 42.4, 40.6, 33.9, 33.4, 32.6, 30.8, 29.3, 29.1, 29.0, 28.3, 27.7, 24.1, 17.3; m/z (EI) 398 (M), 380 (M$\mathrm{H}_{2} \mathrm{O}$ ); HRMS (ESI): [M+Na] $]^{+}$, found 421.2720. $\mathrm{C}_{26} \mathrm{H}_{38} \mathrm{O}_{3} \mathrm{Na}$ requires 421.2713.

4.2.28.

Synthesis

(6bS,8aS,8bS,9S,10aS,11aS,11bR)-9-(4-hydroxy-4-methylpentyl)-8a-methyl-1,2,6b,7,8,8a,9,10,10a,11,11a,11

b-dodecahydro-8bH-cyclobuta[3,4]cyclopenta[1,2-a]phenanthrene-4,8b-diol (cis-22). To a solution of cis-21 
(138 mg, $0.269 \mathrm{mmol})$ in THF $(1.0 \mathrm{~mL})$ was added a $1.0 \mathrm{M}$ solution of TBAF in THF (2.2 mL, $2.2 \mathrm{mmol})$, and the solution was heated under reflux for $55 \mathrm{~h}$. After cooling to room temperature, the resulting mixture was diluted with EtOAc, and washed with water. The aqueous layer was extracted with EtOAc, dried over $\mathrm{Na}_{2} \mathrm{SO}_{4}$, and concentrated in vacuo to give a yellow oil. The crude material was purified by column chromatography (hexane/EtOAc 1:1 to 2:3) to give cis-22 (70.0 mg, 66\%) as a white solid; Mp 222-223 ${ }^{\circ} \mathrm{C}$ (dec.); $[\alpha]_{\mathrm{D}}^{20}+66.9$ (c 1.00, THF); $v_{\max }$ (THF) 3298 (br), 2932, 1616, 1582, 1454, 1369, $1069 \mathrm{~cm}^{-1} ; \delta_{\mathrm{H}}(500$ MHz, DMSO-d $\left.d_{6}\right) 8.98$ (s, 1H), 7.04 (d, $\left.J=8.6 \mathrm{~Hz}, 1 \mathrm{H}\right), 6.50$ (dd, $\left.J=8.3,2.0 \mathrm{~Hz}, 1 \mathrm{H}\right), 6.43$ (d, $J=2.0 \mathrm{~Hz}$, 1H), 4.46 (s, 1H), 4.02 (s, 1H), 2.78-2.65 (m, 2H), 2.29-2.09 (m, 4H), 1.85-1.61 (m, 3H), 1.50-1.13 (m, 14H), 1.04 (s, 6H), 0.67 (s, 3H); $\delta_{\mathrm{c}}\left(125 \mathrm{MHz}, \mathrm{DMSO}-d_{6}\right)$ 154.9, 137.2, 130.6, 126.0, 114.9, 112.7, 86.3, 68.9, 48.8, 45.5, 44.2, 43.6, 40.2, 38.5, 36.4, 31.9, 31.5, 29.5, 29.4, 29.2, 28.8, 27.4, 25.9, 22.1, 14.1; m/z (EI) 398 (M), $380\left(\mathrm{M}-\mathrm{H}_{2} \mathrm{O}\right), 365\left(\mathrm{M}-\mathrm{H}_{2} \mathrm{O}-\mathrm{Me}\right)$; HRMS (ESI): $[\mathrm{M}+\mathrm{H}]^{+}$, found 399.2882. $\mathrm{C}_{26} \mathrm{H}_{39} \mathrm{O}_{3}$ requires 399.2899.

\section{Acknowledgements}

We thank Prof. Hironao Sajiki and Assoc. Prof. Yasunari Monguchi at Gifu Pharmaceutical University for kindly giving us $\mathrm{Pd} / \mathrm{MS} 5 \mathrm{~A}$ and $\mathrm{Pd} / \mathrm{BN}$ as catalysts for chemoselective hydrogenation. This work was 
supported by JSPS KAKENHI grant number 25293002, a Platform for Drug Design, Discovery and Development grant from MEXT and Technology of Japan, and the Hoansha foundation.

\section{Supplementary data}

Copies of ${ }^{1} \mathrm{H}$ and ${ }^{13} \mathrm{C}$ NMR spectra for all new compounds as well as $\mathrm{X}$-ray crystal structures of trans- and cis-22.

\section{References and notes}

(1) For selected examples of small rings used for conformational fixation of biologically active compounds:

(a) Shuto, S.; Ono, S.; Hase, Y.; Ueno, Y.; Noguchi, T.; Yoshii, K.; Matsuda, A. J. Med. Chem. 1996, 39, 4844

-4852. (b) Valasinas, A.; Sarkar, A.; Reddy, V. K.; Marton, L. J.; Basu, H. S.; Frydman, B. J. Med. Chem.

2001, 44, 390-403. (c) Kazuta, Y.; Matsuda, A.; Shuto, S. J. Org. Chem. 2002, 67, 1669-1677. (d) Jarvest,

R.L.; Berge, J. M.; Brown, P.; Houge-Frydrych, C. S. V.; O’Hanlon, P. J.; McNair, D. J.; Pope, A. J.;

Rittenhouseb, S. Bioorg. Med. Chem. Lett. 2003, 13, 1265-1268. (e) Yonezawa, S.; Yamamoto, T.;

Yamakawa, H.; Muto, C.; Hosono, M.; Hattori, K.; Higashino, K.; Yutsudo, T.; Iwamoto, H.; Kondo, Y.;

Sakagami, M.; Togame, H.; Tanaka, Y.; Nakano, T.; Takemoto, H.; Arisawa, M.; Shuto, S. J. Med. Chem. 
2012, 55, 8838-8858. (f) Nakada, K.; Yoshikawa, M., Ide, S.; Suemasa, A.; Kawamura, S.; Kobayashi, T.;

Masuda, E.; Ito, Y.; Hayakawa, W.; Katayama, T.; Yamada, S.; Arisawa, M.; Minami, M.; Shuto, S. Bioorg.

Med. Chem. 2013, 21, 4938-4950.

(2) For selected reviews on the synthesis of small ring compounds: (a) Lebel, H.; Marcoux, J.-F.; Molinaro,

C.; Charette, A. B. Chem. Rev. 2003, 103, 977-1050. (b) Lee-Ruff, E.; Mladenova, G. Chem. Rev. 2003, 103,

1449-1484. (c) Pellissier, H. Tetrahedron 2008, 64, 7041-7095.

(3) (a) Takasu, K.; Ueno, M.; Inanaga, K.; Ihara, M. J. Org. Chem. 2004, 69, 517-521. (b) Takasu, K.; Nagao,

S.; Ueno, M.; Ihara, M. Tetrahedron 2004, 60, 2071-2078. (c) Inanaga, K.; Takasu, K.; Ihara, M. J. Am.

Chem. Soc. 2005, 127, 3668-3669. (d) Takasu, K.; Ishii, T.; Inanaga, K.; Ihara, M. Org. Synth. 2006, 83,

193-199. (e) Takasu, K. Synlett 2009, 1905-1914. (f) Kurahashi, K.; Takemoto, Y.; Takasu, K.

ChemSusChem 2012, 5, 270-273.

(4) Takasu, K.; Nagamoto, Y.; Takemoto, Y. Chem. Eur. J. 2010, 16, 8427-8432.

(5) (a) Abe, E.; Miyaura, C.; Sakagami, H.; Takeda, M.; Konno, K,; Yamazaki, T.; Yoshiki, S.; Suda, T. Proc.

Natl. Acad. Sci. USA 1981, 78, 4990-4994. (b) Lemire, J. M. J. Cell. Biochem. 1992, 49, 26-31. (c) Bouillon,

R.; Okamura, W. H.; Norman, A. W. Endocrine Rev. 1995, 16, 200-257. (d) Haussler, M. R.; Whitfield, G.

K.; Haussler, C. A.; Hsieh, J.-C.; Thompson, P. D.; Selznick, S. H.; Dominguez, C. E.; Jurutka, P. W. J. Bone 
Miner. Res. 1998, 13, 325-349. (e) DeLuca, H. F. Am. J. Clin. Nutr. 2004, 80(suppl.), 1689S-1696S.

(6) Nishii, Y.; Okano, T. Steroids 2001, 66, 137-146.

(7) Carlberg, C.; Mouriño, A. Expert Opin. Ther. Patents 2003, 13, 761-772.

(8) (a) Martínez-Pérez, J. A.; Sarandeses, L.; Granja, J.; Palenzuela, J. A.; Mouriño, A. Tetrahedron Lett.

1998, 39, 4725-4728. (b) Fernández-Gacio, A.; Vitale, C.; Mouriño, A. J. Org. Chem. 2000, 65, 6978-6983.

(c) Varela, C.; Nilsson, K.; Torneiro, M.; Mouriño, A. Helv. Chim. Acta 2002, 85, 3251-3261. (d)

Pérez-García, X.; Rumbo, A.; Larriba, M. J.; Ordóñez, P.; Muñoz, A.; Mouriño, A. Org. Lett. 2003, 5, 4033-

4036. (e) Riveiros, R.; Rumbo, A.; Sarandeses, L. A.; Mouriño, A. J. Org.Chem. 2007, 72, 5477-5485.

(9) Part of this work was published as a preliminary communication: Hata, K.; Arichi, N.; Yamaoka, Y.;

Yamada, K.; Takemoto, Y.; Takasu, K, Asian J. Org. Chem., 2014, 3, 706-710.

(10) (a) Takahashi, A.; Yanai, H.; Taguchi, T. Chem. Commun. 2008, 2385-2387. (b) Yanai, H.; Takahashi,

A.; Taguchi, T. Chem. Commun. 2010, 46, 8728-8730.

(11) The most stable conformer of cis- and trans-3b was calculated at the B3LYP/6-31G* level. Gaussian 98,

Revision A.6, M. J. Frisch, G. W. Trucks, H. B. Schlegel, G. E. Scuseria, M. A. Robb, J. R. Cheeseman, V. G.

Zakrzewski, J. A. Montgomery, Jr., R. E. Stratmann, J. C. Burant, S. Dapprich, J. M. Millam, A. D. Daniels,

K. N. Kudin, M. C. Strain, O. Farkas, J. Tomasi, V. Barone, M. Cossi, R. Cammi, B. Mennucci, C. Pomelli, C. 
Adamo, S. Clifford, J. Ochterski, G. A. Petersson, P. V. Ayala, Q. Cui, K. Morokuma, D. K. Malick, A. D.

Rabuck, K. Raghavachari, J. B. Foresman, J. Cioslowski, J. V. Ortiz, B. B. Stefanov, G. Liu, A. Liashenko, P.

Piskorz, I. Komaromi, R. Gomperts, R. L. Martin, D. J. Fox, T. Keith, M. A. Al-Laham, C. Y. Peng, A.

Nanayakkara, C. Gonzalez, M. Challacombe, P. M. W. Gill, B. Johnson, W. Chen, M. W. Wong, J. L. Andres,

C. Gonzalez, M. Head-Gordon, E. S. Replogle, and J. A. Pople, Gaussian, Inc., Pittsburgh, PA, 1998.

(12) Hutchins, R. O.; Kandasamy, D.; Dux, F., III; Maryanoff, C. A.; Rotstein, D.; Goldsmith, B.; Burgoyne,

W.; Cistone, F.; Dalessandro, J.; Puglis, J. J. Org. Chem. 1978, 43, 2259-2267.

(13) Takahashi, T.; Yoshimura, M.; Suzuka, H.; Maegawa, T.; Sawama, Y.; Monguchi, Y.; Sajiki, H.

Tetrahedron 2012, 68, 8293-8299.

(14) (a) Yabe, Y.; Yamada, T.; Nagata, S.; Sawama, Y,; Monguchi, Y.; Sajiki, H. Adv. Synth. Catal. 2012,

354, 1264-1268. (b) Yabe, Y.; Sawama, Y.; Yamada, T.; Nagata, S.; Monguchi, Y.; Sajiki, H.

ChemCatChem 2013, 5, 2360-2366. (c) Kawanishi, A.; Miyamoto, C.; Yabe, Y.; Inai, M.; Asakawa, T.;

Hamashima, Y.; Sajiki, H.; Kan, T. Org. Lett. 2013, 15, 1306-1309.

(15) (a) Jorgensen, M. J. J. Am. Chem. Soc. 1969, 91, 6432-6443. (b) Al Dulayymi, J. R.; Baird, M. S.; Jones,

K. Tetrahedron 2004, 60, 341-345.

(16) (a) Node, M.; Ohta, K.; Kajimoto, T.; Nishide, K.; Fujita, E.; Fuji, K. Chem. Pharm. Bull. 1983, 31, 
4178-4180. (b) Foucher, V.; Guizzardi, B.; Groen, M. B.; Light, M.; Linclau, B. Org. Lett. 2010, 12, 680683.

(17) (a) Ireland, R. E.; Walba, D. M. Org. Synth. 1977, 56, 44. (b) Peters, R. H.; Crowe, D. F. ; Avery, M. A. ;

Chong, W. K. M.; Tanabe, M. J. Med. Chem. 1989, 32, 1642-1652.

(18) Passiniemi, M.; Koskinen, A. M. P. Org. Biomol. Chem. 2011, 9, 1774-1783. 